\title{
NDC1 is necessary for the stable assembly of the nuclear pore scaffold to establish nuclear transport in early C. elegans embryos
}

Michael Sean Mauro ${ }^{1}$, Gunta Celma ${ }^{1}$, Vitaly Zimyanin ${ }^{2,3}$, Kimberley H. Gibson ${ }^{4}$, Stefanie Redemann $^{2,3,5}$, and Shirin Bahmanyar ${ }^{1,6, *}$

${ }^{1}$ Department of Molecular, Cellular and Developmental Biology, Yale University, New Haven, CT 06511, USA

${ }^{2}$ Center for Membrane and Cell Physiology, University of Virginia, School of Medicine, Charlottesville, VA, USA

${ }^{3}$ Department of Molecular Physiology and Biological Physics, University of Virginia, School of Medicine, Charlottesville, VA, USA

${ }^{4}$ Center for Cellular and Molecular Imaging: Electron Microscopy, Department of Cell Biology, Yale School of Medicine, New Haven, CT, USA

${ }^{5}$ Department of Cell Biology, University of Virginia, School of Medicine, Charlottesville, VA, USA

${ }^{6}$ Lead contact

"Corresponding author: shirin.bahmanyar@yale.edu

Keywords: Ndc1; nuclear pore complex; nuclear envelope; mitosis; Nup107-160 complex; Nup53; post-mitotic NPC assembly; outer ring scaffold; $C$. elegans embryos 


\section{Summary}

Nuclear pore complexes (NPCs) are large protein assemblies that facilitate transport of macromolecules across the nuclear envelope (NE) [1, 2]. How thousands of NPCs rapidly assemble after open mitosis to form a functional NE is not known. Recruitment of the Nup107160 outer ring scaffold to chromatin initiates NPC assembly. The Nup53/93 complex bridges the outer ring to the central channel to form a functional pore [3-6]. Nup53 interacts with the conserved transmembrane nucleoporin Ndc1; however, how Ndc1 contributes to post-mitotic NPC assembly is unclear [7-9]. Here, we use C. elegans embryos to show that the timely formation of a functional $\mathrm{NE}$ after mitosis depends on Ndc1. Endogenously tagged Ndc1 is recruited early to the reforming $\mathrm{NE}$ and is highly mobile in the nuclear rim. 3D analysis of post-mitotic NE formation revealed a decreased NPC density in NEs of ndc1 deleted embryos - continuous nuclear membranes contained few holes where assembling NPCs are normally located. Nup160 is highly mobile in NEs depleted of Ndc1 and outer ring scaffold components are less enriched at the rim. When both ndc1 and nup53 are absent, nuclear assembly fails. Together, these data show that Ndc1 dynamically associates with the NE and promotes stable association of the outer ring scaffold in the NE to facilitate NPC assembly after open mitosis. Furthermore, Ndc1 and Nup53 function in parallel to drive nuclear assembly. We propose that Ndc1 is a dynamic membrane adaptor that helps recruit and promote the self-assembly of the nuclear pore scaffold to drive post-mitotic NPC assembly. 


\section{Results and Discussion}

A previous genome-wide high content RNAi-screen using differential interference contrast (DIC) to film early C. elegans embryos showed that RNAi-depletion of ndc1 results in pronuclei that are smaller in size than in control embryos [10]. We used CRISPR-Cas9 gene editing to delete the $n d c 1$ gene locus and eliminate ndc1 mRNA expression (Figure S1A and S1B; ndc1A). ndc1 $\Delta$ embryos contained small pronuclei (Figure 1B) that were similar in size to those RNAidepleted for $n d c 1$ (Figure S1C). Embryos produced from homozygous $n d c 1 \Delta$ worms had a range of lethality $(40 \%-100 \%$, average \pm S.D. $=61 \% \pm 21$, Figure $1 \mathrm{C})$, that was similar but more severe than embryos produced from a mutant strain of $n d c 1\left(n d c-1^{t m 2886}\right)$ in which $50 \%$ of the $n d c 1$ gene coding region is deleted (range $10 \%-80 \%$, average \pm S.D. $=48 \% \pm 25 \%$, Figure S1D and S1E; [11]). The size of $n d c 1 \Delta$ embryos, but not ndc1 RNAi-depleted embryos, appeared smaller suggesting a potential defect in germline development that may explain the embryonic lethality that was not observed by RNAi-depletion of ndc1 (Figure 1B, S1C, and S1F). The fact that both $n d c 1 \Delta$ and RNAi-depletion of $n d c 1$ consistently result in small pronuclei suggested that $\mathrm{Ndc1}$ is necessary for a process that controls nuclear size in early embryos.

The rate of nuclear import predicts the rate of nuclear expansion and nuclear size [12-15]. The rapid and stereotypical early divisions of $C$. elegans embryos provides an opportunity to quantitatively monitor nuclear import from the onset of nuclear formation until nuclear permeabilization in mitosis (Figure 1D; [16]). Fertilization of the oocyte by haploid sperm initiates two rounds of meiotic chromosome segregation. The sperm chromatin is devoid of a nuclear envelope (NE) at the time of fertilization. After anaphase of meiosis II, components in the oocyte cytoplasm are recruited to the sperm-derived pronucleus to form a functional NE (Figure 1D). Transport competent pronuclei then expand $\sim 30$-fold in volume prior to the onset of nuclear permeabilization, which follows pronuclear meeting and regression of the pseudocleavage (Figure 1D; [17]). Nuclear import monitored by a GFP reporter fused to a nuclear localization 
(GFP:NLS-Lacl, hereafter referred to as GFP:NLS) shows nuclear accumulation of GFP fluorescence throughout the time course of nuclear expansion (Figure 1D-1F). RNAi-depletion of Ndc1 results in lower levels of nuclear accumulation of the GFP:NLS reporter (Figure 1E-1F, Video S1). Nuclear import is more severely impaired in embryos depleted of the nuclear basket component Nup153 (npp-7) and the essential inner ring component Nup53 (npp-19) (Figure 1E1F, Video S1; [18-22]). The average diameter of pronuclei is $\sim 8 \mu \mathrm{m} \sim-100 \mathrm{~s}$ relative to pseudocleavage regression in control embryos (Figure 1G) and the diameter of pronuclei at the same timepoint in each RNAi condition scales with the degree of severity in defective nuclear import for that condition (6.2 $\mu \mathrm{m}$ for $n d c 1(R N A i)$ versus $5.3 \mu \mathrm{m}$ for nup153(RNAi) and $3.8 \mu \mathrm{m}$ for nup53(RNAi); Figure1F and 1G). Thus, reduced nuclear import likely causes smaller pronuclei to form in the absence of Ndc1 suggesting that Ndc1 serves a specific function to promote nuclear transport in early C. elegans embryos.

To determine if Ndc1 promotes nuclear transport by contributing to the assembly of the NE, we first analyzed the dynamics and localization of Ndc1 during the first mitotic division. Ndc1

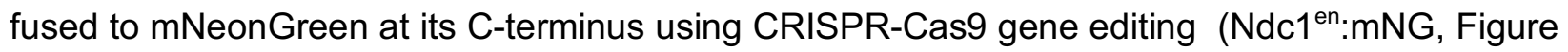
$\mathrm{S} 2 \mathrm{~A}$ and $\mathrm{S} 2 \mathrm{~B}$ ) is enriched at the NE and appears functional given the normal pronuclear size in these embryos (Figure S2B, S2C, and Video S2). $\mathrm{Ndc}^{\mathrm{en}}$ :mNG also localizes to the ER as well as punctate structures throughout the cytoplasm that disperse into the ER upon entry into mitosis (Figure S2C). Upon mitotic exit, $\mathrm{Ndc} 1^{\mathrm{en}}: \mathrm{mNG}$ accumulates at the reforming NE with similar kinetics as the inner NE protein LEM-2 (LEM-2:mCh; Figure 2A, 2B, and Video S3) and coincident with the appearance of signal from the fluorescent ER maker SP12:GFP around segregated chromosomes (Figure S2D). The early recruitment of Ndc1 to the post-mitotic NE suggested a potential role for $\mathrm{Ndc1}$ in establishment of nuclear transport.

We next analyzed the kinetics of NE formation relative to the establishment of nuclear transport directly after anaphase onset. The NE rapidly forms around daughter nuclei $\sim 100 \mathrm{~s}$ after 
anaphase onset, coincident with the ingression of the cytokinetic furrow and followed by expansion of daughter nuclei (Figure 2A and 2C). In control embryos, the GFP:NLS fluorescence signal appears in the nucleus $\sim 40 \mathrm{~s}$ following initiation of furrow ingression, which is $\sim 60-80 \mathrm{~s}$ following the initial presence of a complete nuclear rim marked by LEM2:mCh (-20 s, Figure 2C). GFP:NLS continues to accumulate in the nucleus as nuclei expand (Figure $2 \mathrm{C}$ ). In the absence of $n d c 1$, LEM-2:mCh forms a nuclear rim with normal timing, however the GFP:NLS fluorescence signal is only detectable in the daughter pronuclei at $\sim 120 \mathrm{~s}$ following initiation of furrow ingression, which is $\sim 80-100$ s later than in control embryos (Figure 2D). In addition to the later appearance of nuclear GFP, the nuclear to cytoplasmic ratio of the GFP:NLS fluorescence signal (see Figure 1F) was lower in ndc1 RNAi-depleted embryos throughout the rest of the cell cycle (Figure 2E, Video S4).

To examine if the decreased levels of nuclear GFP:NLS fluorescence signal after anaphase onset in ndc1 RNAi-depleted embryos (Figure 2E and $2 \mathrm{~F}$ ) result from a delay in establishment of nuclear transport, we normalized each individual trace of the nuclear to cytoplasmic ratio to its maximum value (Figure S2E). These data revealed a shift in the onset of nuclear accumulation of GFP:NLS in ndc1 RNAi-depleted embryos (Figure S2E). Plotting the difference between each time point for the average normalized fluorescence intensities further revealed a slower rate of change in the nuclear accumulation of GFP:NLS after anaphase onset in ndc1 RNAi-depleted embryos (Figure 2G). These data indicate that loss of Ndc1 delays nuclear import upon NE reformation.

The early recruitment of Ndc1 to the nascent post-mitotic NE combined with the delay in nuclear import resulting from loss of $\mathrm{Ndc1}$ indicates that $\mathrm{Ndc1}$ is necessary for the timely establishment of nuclear transport upon NE formation.

To determine how Ndc1 establishes nuclear transport upon NE formation, we analyzed serial sections of embryos from electron tomograms of NE formation processed at the initiation of furrow ingression (Figure 3A and 3B; Videos S5, S6 and S7). We focused on nascent nuclear 
membranes wrapped around the outer edges of chromatin where NPCs first assemble, also known as the "non-core" region [23]. 3D analysis of the reforming NE revealed small and large gaps at this time point and those $<100 \mathrm{~nm}$ were marked as potential NPCs (Figures 3A, 3B, S3A, and S3B; Videos S8 and S9; [5]). The NE in ndc1 $\Delta$ embryos was mostly continuous and contained an average of 8.6 "NPC" holes per $\mu \mathrm{m}^{2}$ ( $n=3$ areas) (Figure 3B, S3B and Video S9). In contrast, the NE in a control embryo contained an average of 37 "NPC" holes per $\mu m^{2}(\mathrm{n}=2$ areas) and was more discontinuous (Figure 3A and 3C; Video S8). Thus, when ndc1 is absent, nascent NEs of the "non-core" region are more continuous and on average contain $\sim 4.3$ fold fewer holes that fit the dimensions of nascent NPCs [5]. These data together with the slower rate of nuclear import resulting from loss of Ndc1 suggested that Ndc1 promotes NPC assembly during NE formation. The lower density of NPCs may limit the rate of accumulation of nuclear cargo during early stages of NE formation since soluble cargo are also lost by diffusion across larger gaps that are not yet sealed [24].

The outer ring scaffold is essential for forming NEs that contain NPCs [6] and so we assessed whether loss of Ndc1 affects the localization of members of the outer scaffold complex (also known as the Y-complex [1, 2], Figure 3D). In early embryos, the Y-complex component Nup160:GFP localizes to kinetochores and to the nuclear rim (Figure S3C; [25]). Nup160:GFP also localizes to puncta throughout the cytoplasm that occasionally co-localize with Ndc1 puncta and disperse upon entry into mitosis (Figure S3D and S3E). ndc1 RNAi-depleted embryos contained lower levels of Nup160:GFP at the nuclear rim (Figure 3D) and very few puncta formed in the cytoplasm (Figure S3E). Nup160:GFP puncta may represent preassembled nucleoporins known to be abundant in early embryos that contain excess nucleoporins [26]. The fact that they co-localize with Ndc1 and do not form in ndc1 RNAi embryos, which also have lower levels of Nup160:GFP at the nuclear rim, suggested that Ndc1 may play a role in stabilizing scaffold components both in these cytoplasmic structures and in the NE. 
The reduced nuclear rim signal of Nup160:GFP resulting from loss of $n d c 1$ likely reflects the lower density of NPCs in the NE. In addition to Nup160:GFP, line profiles of fluorescence intensities of GFP fusions to Nup133 ${ }^{\mathrm{NPP}-15}$, Seh1 $1^{\mathrm{NPP}-18}$, Nup85 $5^{\mathrm{NPP}-2}$, and Elys ${ }^{\mathrm{MEL}-28}$ revealed that each component is approximately $50 \%$ less enriched at the nuclear rim in the absence of Ndc1 (Figure 3D; [25]). Immunostaining with antibodies that recognize Nup $107^{\text {NPP-5 }}$ and Elys ${ }^{\text {MEL-28 }}$ confirmed lower endogenous levels of $\mathrm{Y}$-complex nucleoporins at the nuclear rim in $n d c 1 \Delta$ early embryos (Figure S3F and S3G; [27, 28]). The total protein levels of Nup107 in ndc1 $\Delta$ worms were similar to control worms (Figure $\mathrm{S} 3 \mathrm{H}$ ). Deletion of ndc1 also results in lower levels of the endogenous inner ring component Nup53 at the nuclear rim (Figure S4A; [19]), but not its global protein levels (Figure S4B). Immunostaining of control and ndc1 mutant worms with mAB414, a monoclonal antibody that recognizes FG-nucleoporins, did not show a consistent difference in fluorescence signal at the nuclear rim in early stage ndc1 mutant embryos (Figure S4C), although prior work in late-stage $C$. elegans embryos showed that loss of $n d c 1$ decreases the levels of FG nucleoporins at the NE [11]. Total protein levels of mAB414 epitope-containing nucleoporins and the FG nucleoporins Nup96/98 was unchanged (Figure S4D and S4E; ). Together, these results provide further evidence that Ndc1 is involved in assembly of the NPCs at the NE in early $C$. elegans embryos.

The fact that Ndc1 is an integral membrane protein localized to the pore membrane has led to the proposal that it serves as a membrane anchor for NPCs [8], although currently direct evidence for this does not exist. Fluorescence recovery after photobleaching (FRAP) revealed that $\sim 80 \%$ of the Nup160:GFP pool in the NE is immobile in control embryos (Figure 3E and Video S10; average mobile fraction $\pm S D=0.20 \pm 0.06, n=8$ nuclei), similar to what has been shown in mammalian cells [29]. In ndc1 RNAi depleted embryos there was a greater than 2-fold increase in the mobile fraction of Nup160:GFP in the NE indicating that Nup160:GFP is less stably incorporated without Ndc1 (Figure 3E; average mobile fraction $\pm \mathrm{SD}=0.47+/-0.12, \mathrm{n}=8$ nuclei). 
We reasoned that if Ndc1 serves as an anchor to the NPC to immobilize Nup160:GFP, then Ndc1 itself should be immobile at the NE. Instead, we found that $\mathrm{Ndc}^{\mathrm{en}}$ :mNG is highly mobile in the NE (Figure 3F and Video S11; average mobile fraction $\pm S D=0.59 \pm-0.09, t_{1 / 2}$ of $56.8 \pm 26.3 \mathrm{~s}, \mathrm{n}=8$ embryos). This data is in line with recent data using metabolic labeling in budding yeast showing that $\mathrm{Ndc1}$, unlike other transmembrane nucleoporins, is readily exchanged in the NPC [30]. Thus, Ndc1 may function as a dynamic membrane adaptor that promotes the stable association of outer ring scaffold components in the NE.

Ndc1 binds directly to Nup53, which links the inner and outer rings of the NPC to the central channel through its association with the Nup96/98 complex and Nup155 (Figure 4A and S4F; $[7-9,31,32])$. Prior work had suggested that Ndc1 recruits Nup53 to the assembling NPC [8] and that Ndc1 functions in NPC assembly through its interaction with Nup53 [7-9]. To determine if Ndc1 and Nup53 cooperate in post-mitotic NPC assembly in C. elegans, we utilized a strain carrying a partially functional mutant allele of Nup53 (nup53 $\left.3^{t m 2886}\right)$ that is missing a central region important for its dimerization (Figure S4G; [19]). The mutant Nup53( $\Delta$ aa 217-286) protein is expressed at lower levels compared to wild type Nup53 (Figure S4B; [19]) and on average $50 \%$ of embryos produced from homozygous nup $53^{\text {tm2886}}$ worms can survive to hatching, as has been shown previously (Figure 4B; [19]). Live imaging of nup53 ${ }^{\text {tm2886 }}$ one-cell embryos revealed that sperm pronuclei expand and establish import (Figure 4C), similar to nup53 RNAi-depleted nuclei (Figure 1E-1G) but to a lesser extent than control and ndc1 RNAi-depleted embryos (Figure $1 \mathrm{E}-1 \mathrm{G})$. RNAi-depletion of $n d c 1$ strongly enhanced embryonic lethality in the nup $53^{\text {tm}} 2886$ strain (Figure 4B) - these embryos completely failed to assemble a NE around sperm chromatin (Figure 4C and 4D). Sperm chromatin in nup53 $3^{\text {tm2886 }}$ mutant embryos depleted of ndc1 remains compacted and nuclear import is never established, even at -100 s relative to $P C$ regression when GFP:NLS accumulates to detectable levels in the sperm-derived pronucleus of nup53 ${ }^{\text {tm2886 }}$ embryos (Figure 4C and 4E). 
In nup53 ${ }^{\text {tm2886 }}$ mutant and nup53 RNAi-depleted embryos, Nup160:GFP appears patchy at the sperm pronuclear rim indicating an abnormal distribution of NPCs (Figure 4D and S4H). In contrast, in nup53 ${ }^{\operatorname{tm} 2886}$ embryos RNAi-depleted of ndc1, the Nup160:GFP signal persisted on chromatin (Figure 4D) and the nuclear diameter at -100 s prior to PC regression was significantly smaller (Figure 4E; average diameter of $2.6 \mu \mathrm{m}$ versus $\sim 4 \mu \mathrm{m}$ in nup $53^{\text {tm } 2886}$ or $n d c 1$ (RNAi) only conditions) indicating a failure in nuclear formation. These data show that Ndc1 is necessary for the recruitment of Nup160:GFP to the nuclear rim and assembly of nuclei, albeit in a defective manner, when Nup53 function is compromised.

Failure to assemble a NE was also observed in fixed ndc1 $1 \Delta$ embryos RNAi-depleted for nup53 (Figure 4F-4H). The mAB414 signal around chromatin was normal in ndc1 $\Delta$ embryos and patchy in $100 \%$ of fixed one-cell stage embryos RNAi-depleted for nup53 alone (Figure $4 \mathrm{H}$ and Figure S4I). The patchy Nup160:GFP (Figure 4D and S4H) and mAB414 (Figure 4H and S4I) signal resulting from loss of Nup53 suggests aberrant assembly of NPCs. Surprisingly, these NEs still support some nuclear import and expansion (Figure 4D, 4E and Figure 1E-1G). One-cell stage ndc1 1 embryos RNAi-depleted for nup53 contained highly compacted sperm chromatin (Figure 4F and 4G) with little to no mAB414 signal surrounding the chromatin mass (Figure 4F and $4 \mathrm{H}$ ). Together, these data provide evidence that Ndc1 and Nup53 function, at least in part, in parallel pathways to drive robust nuclear assembly in early C. elegans embryos.

Cumulatively, the results above demonstrate that Ndc1 is recruited early to the reforming NE after mitosis where it dynamically associates with the NE and serves to stabilize the outer ring scaffold to drive NPC assembly (Figure 4I). Our in vivo genetic analyses further demonstrate that the loss of $n d c 1$ strongly enhances the NE assembly defect resulting from loss of Nup53 (and vice versa) indicating that Ndc1 and Nup53 coordinate the assembly of a functional NE (Figure 4I).

We suggest that Ndc1 functions as a dynamic membrane adaptor that helps recruit and orient scaffold nucleoporins at membrane holes to promote their self-assembly and drive 
formation of NPCs. Electron tomography studies of the earliest stages of NE reformation revealed that assembly of nuclear pores initiates in $40 \mathrm{~nm}$ NE holes that then dilate upon scaffold assembly [5]. Interestingly, recent work has shown that preassembled Y-complex subunits associated with holes in the ER initiate NPC assembly after anaphase [33]. The fact that loss of $n d c 1$ results in nascent NEs that are continuous but with only a few holes $<100 \mathrm{~nm}$ suggests that Ndc1 may be necessary for sculpting or maintaining these small holes that are for NPC assembly. Furthermore, the cytoplasmic puncta we observed with $\mathrm{Ndc1}$ may be annulate lamellae, specialized membranes in the ER that are prominent in embryos and contain symmetric scaffold nuclear pores to store excess nucleoporins [26]. The fact that Nup160 cytoplasmic puncta sometimes colocalize with $\mathrm{Ndc1}$ puncta in the ER and no longer form without Ndc1 suggests that Ndc1 may be involved in formation of these structures. However, we show that Ndc1 is highly dynamic in the membrane arguing against a role as a structural protein or immobile anchor at the pore membrane. More work needs to be done to understand the identity of these cytoplasmic puncta, their mechanistic relationship to Ndc1, and whether they are directly involved in NPC assembly in C. elegans embryos.

Given that there are still NPCs assembled in the absence of Ndc1, albeit at a significantly decreased density, indicates that scaffold nucleoporins can assemble without $\mathrm{Ndc1}$, but perhaps at a slower rate. It is also possible that NPCs can also assemble through a parallel pathway to Ndc1. Supporting this idea, our work and work in other systems show that loss of Ndc1 and Nup53 inhibits NPC assembly [7-9, 21]. Nup53 has been shown to bind directly to membranes [21] and a region of Ndc1 that does not bind to Nup53 supports NPC assembly when Nup53's membrane association region is deleted in in vitro assembled nuclei using Xenopus extracts [7]. This suggests that Ndc1 can drive NPC assembly independently of its interaction with Nup53 and supports our working model that these proteins function at least in part in parallel pathways to drive post-mitotic NPC assembly (Figure 4I). Future is required to determine how Ndc1 acts to 
immobilize the outer ring scaffold in the NE and how its role at the membrane coordinates with Nup53 to enable nuclear assembly.

\section{Acknowledgements}

We thank Sarah Barger for helpful feedback on the manuscript and members of the Bahmanyar lab for helpful discussion. We thank Eric Hastie and Sarah Barger for help with cloning, the MBL Embryology course for insights into strain construction, Jackson Gordon for help with FRAP analysis. We thank Peter Askajar for sharing antibodies against various C elegans Nups, Arshad Desai, the Caenorhabditis Genetics Center and the Japanese knockout (KO) consortium for strains utilized in this study. This work was supported by an NSF CAREER Award to Shirin Bahmanyar (NSF CAREER 1846010), and NIH CMB Training Grant: T32GM007223-S1to Michael Mauro.

\section{Author Contributions}

M.S. Mauro and S. Bahmanyar conceived the project. M.S. Mauro performed the majority of the experiments. G. Celma generated and filmed fluorescent strains used in this manuscript. V. Zimyanin and S. Redemann prepared samples by high-pressure freezing, generated electron tomograms, and joined the mutant tomogram. K.H. Gibson joined the wild type tomograms. M. S. Mauro generated 3D models of reconstructed tomograms. M.S. Mauro and S. Bahmanyar wrote the manuscript, with input from all authors. S. Bahmanyar supervised the project.

The authors declare no competing interests 
A

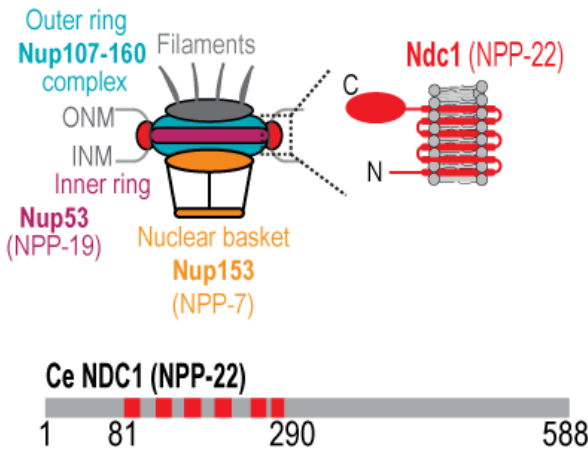

B

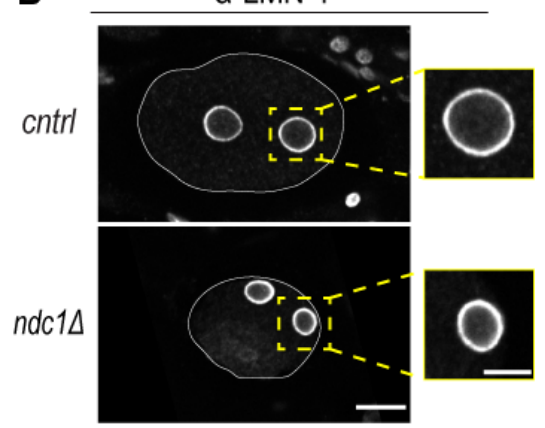

Mauro Figure 1

C

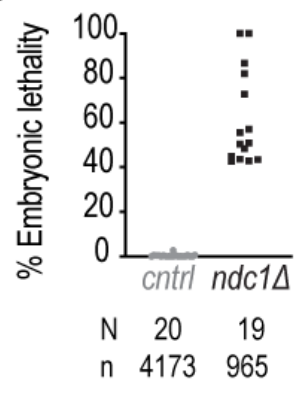

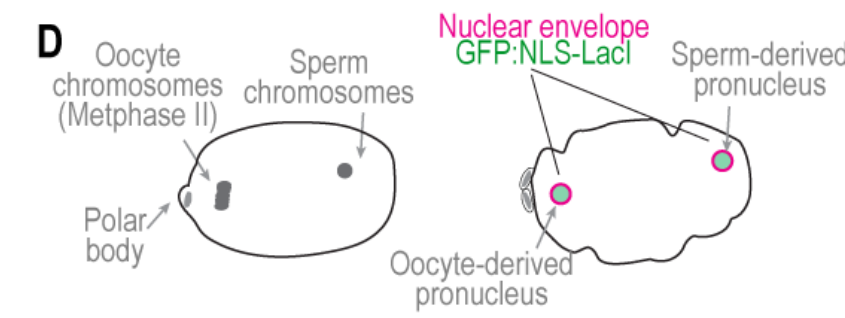

Time (s) relative pronucleus

to $\mathrm{PC}$ regression $-500 s$ $-400 s$

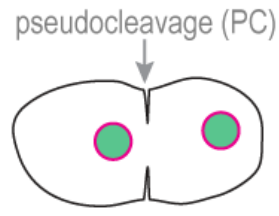
regression

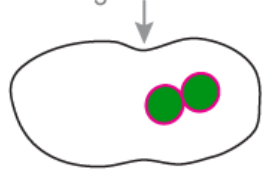

E

Time (s) relative to $\mathrm{PC}$ regression
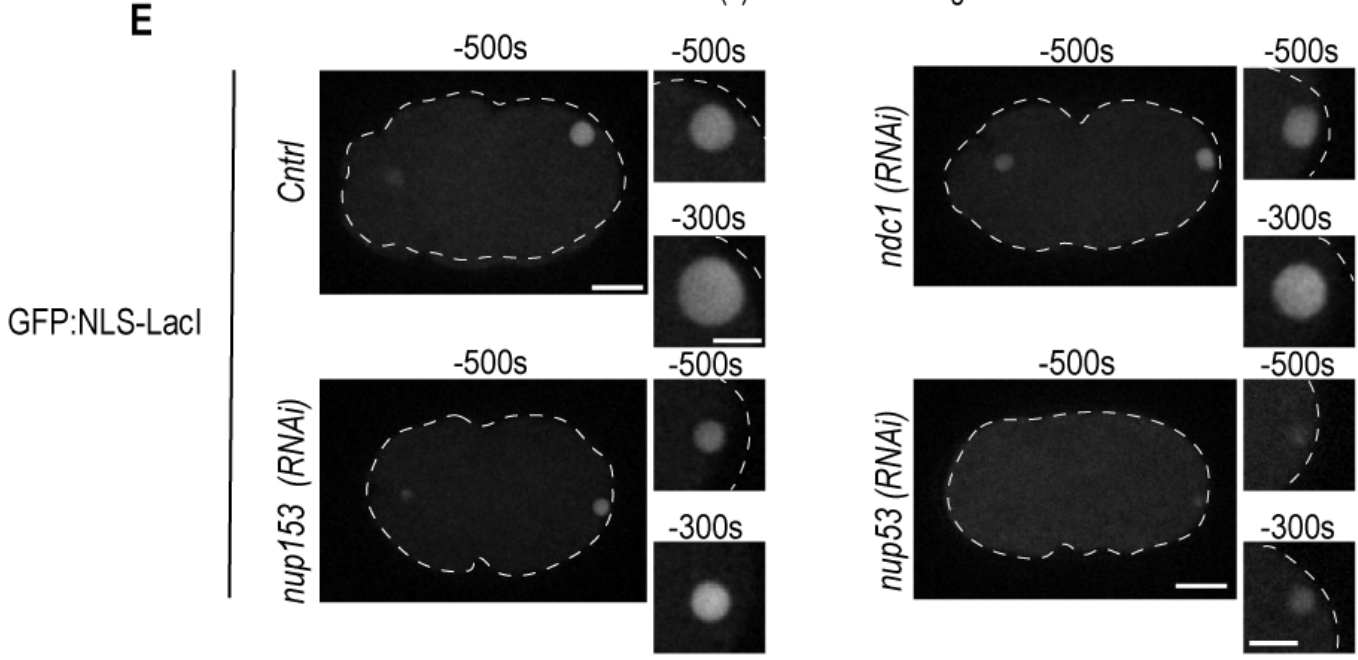

\section{F}

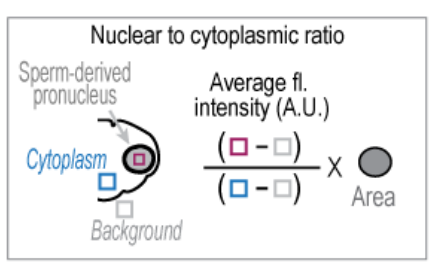

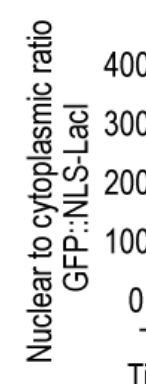

Time $(s)$

nup153 (RNAi), $n=7 \quad$ nup53 (RNAi), $n=8$
G $\quad-100$ s relative to $\mathrm{PC}$ regression

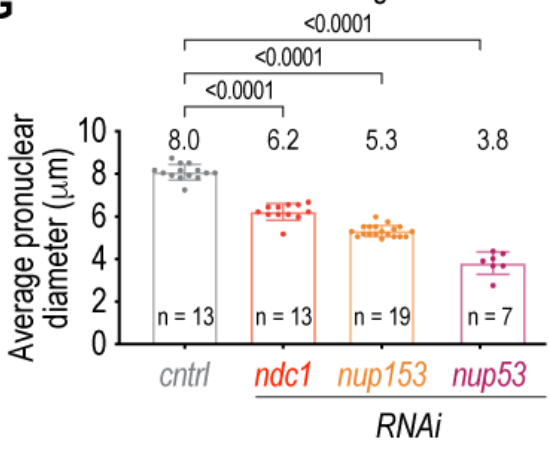


Figure 1. Smaller pronuclear size resulting from loss of Ndc1 corresponds to reduced nuclear import in early C. elegans embryos. (A) Schematic of an NPC (left). Schematic and domain organization of $\mathrm{Ce} \mathrm{Ndc1} \mathrm{(right,} \mathrm{bottom)} \mathrm{(B)} \mathrm{Fixed} \mathrm{overview} \mathrm{and} \mathrm{magnified} \mathrm{images} \mathrm{of} C$. elegans embryos immunostained for lamin for indicated conditions. Scale bars, $10 \mu \mathrm{m}$ and $5 \mu \mathrm{m}$ for magnified images. (C) Plot of percentage embryonic lethality for indicated conditions. $\mathrm{N}=\#$ of worms. $n=$ number of embryos. (D) Schematic of stereotypical nuclear events relative to nuclear import in early embryos in the $C$. elegans zygote, with pseudocleavage (PC) regression used as a reference time point. Time is in seconds. (E) Confocal overview and magnified images of embryo from a time lapse series of GFP:NLS-Lacl in indicated conditions. Scale bars, $10 \mu \mathrm{m}$ for overview image and $5 \mu \mathrm{m}$ for magnified images. (F) Schematic for equation to calculate nuclear to cytoplasmic ratio of GFP::NLS-Lacl (left) and corresponding plot for indicated conditions (right). Average \pm S.D. is shown. (G) Pronuclear diameter for indicated conditions at indicated timepoint. Average \pm S.D. is shown. $n=\#$ of embryos. A two-way ANOVA was used to determine statistical significance between control and each RNAi condition. p-values all $<0.0001$. 
Mauro Figure 2
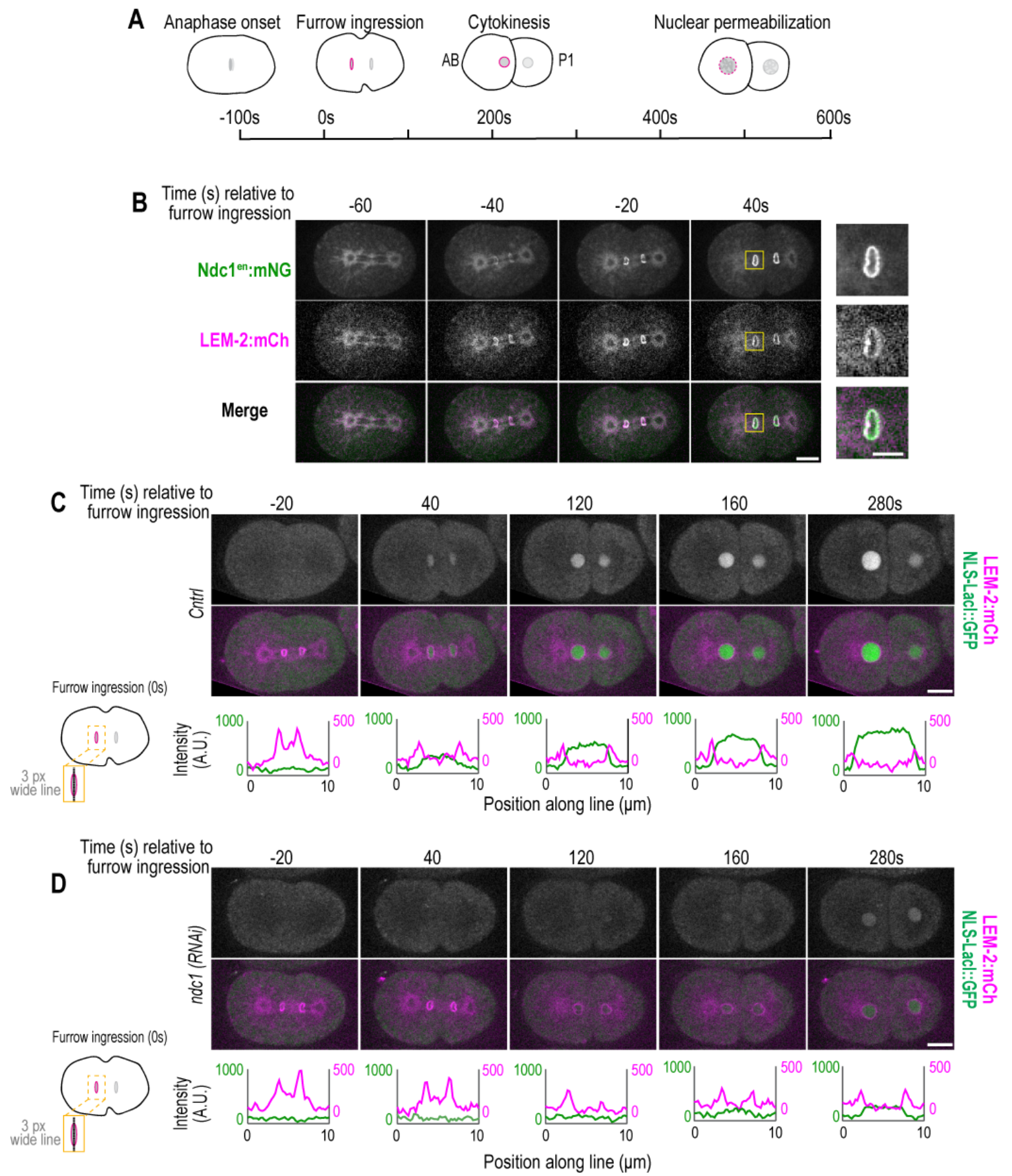

E

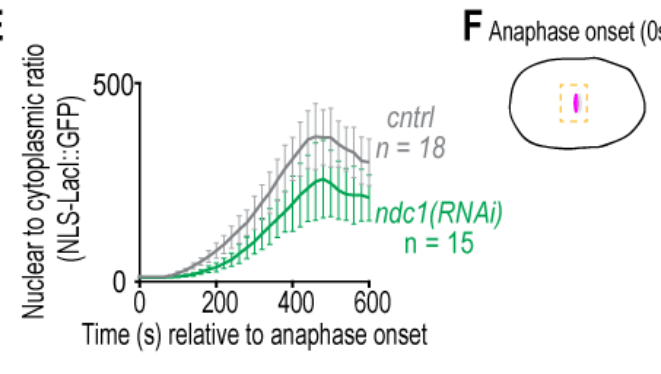

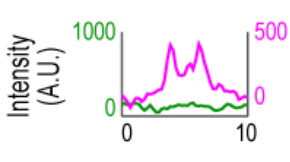
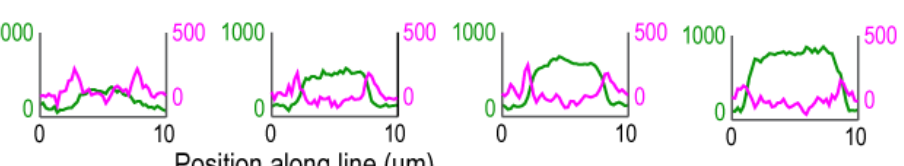

.

Position along line $(\mu \mathrm{m})$ 
Figure 2. Ndc1 is necessary for timely formation of a transport competent nucleus after mitosis. (A) Schematic of the first mitotic division in C. elegans embryos elative to anaphase onset and initiation of furrow ingression. All measurements of 1 to 2 cell stage embryos are done on the $A B$ nucleus (NE is highlighted magenta). (B) Confocal images from time series of mitotic nuclear formation relative to furrow ingression with indicated markers. (C-D) (Above) Confocal images from time series mitotic nuclear formation with indicated markers for indicated conditions. (Below) Line scans measuring background-corrected fluorescent intensities as indicated in schematic for each time point and fluorescent marker. Scale bars, $10 \mu \mathrm{m}$ and $5 \mu \mathrm{m}$ for magnified images. (E) Plot of nuclear to cytoplasmic ratio of GFP:NLS-Lacl for indicated conditions. $n=$ \# of embryos. Average \pm S.D. is shown. (F) Confocal images of chromosome region from time series relative to anaphase onset with indicated markers and in indicated conditions. Scale bar, $5 \mu \mathrm{m}$. (G) Equation and plot of rate of change in the nuclear to cytoplasmic ratio of GFP:NLS-Lacl between each time point in the first $200 \mathrm{~s}$ after anaphase for the indicated conditions. Average is shown. 

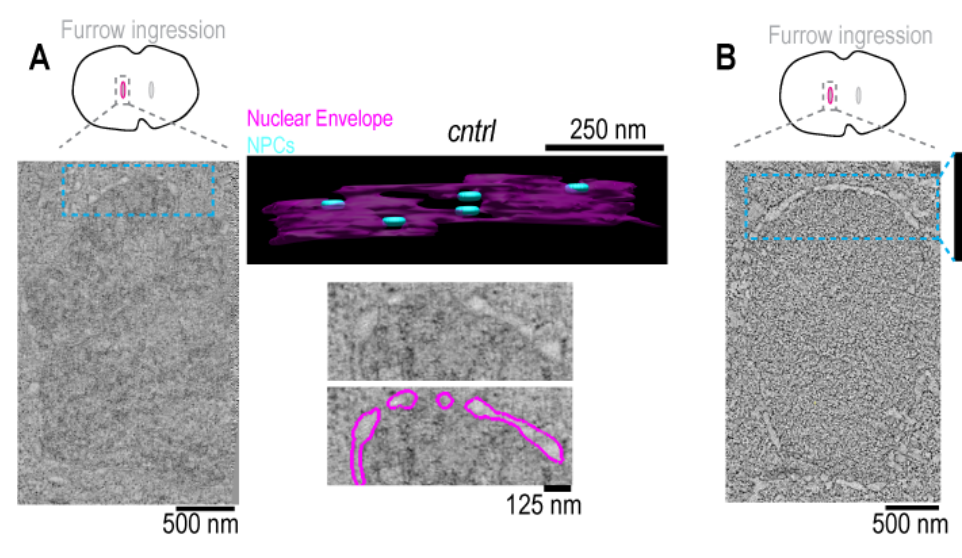

Mauro Figure 3

D

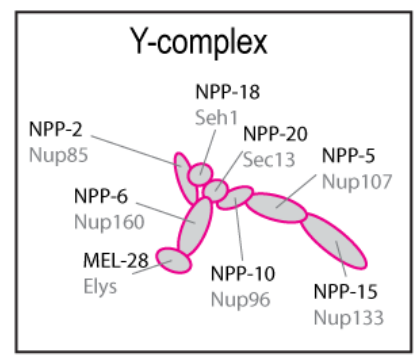

$300 \mathrm{~s}$ post anaphase onset

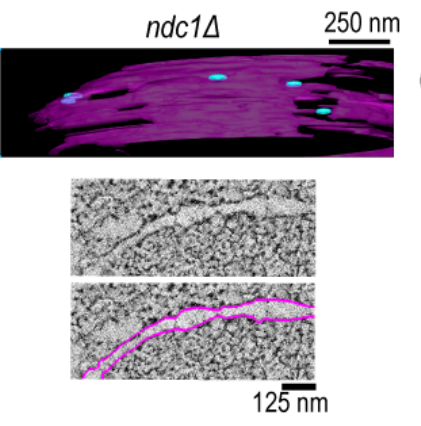

\section{C}

Diameter of holes $<100 \mathrm{~nm}$
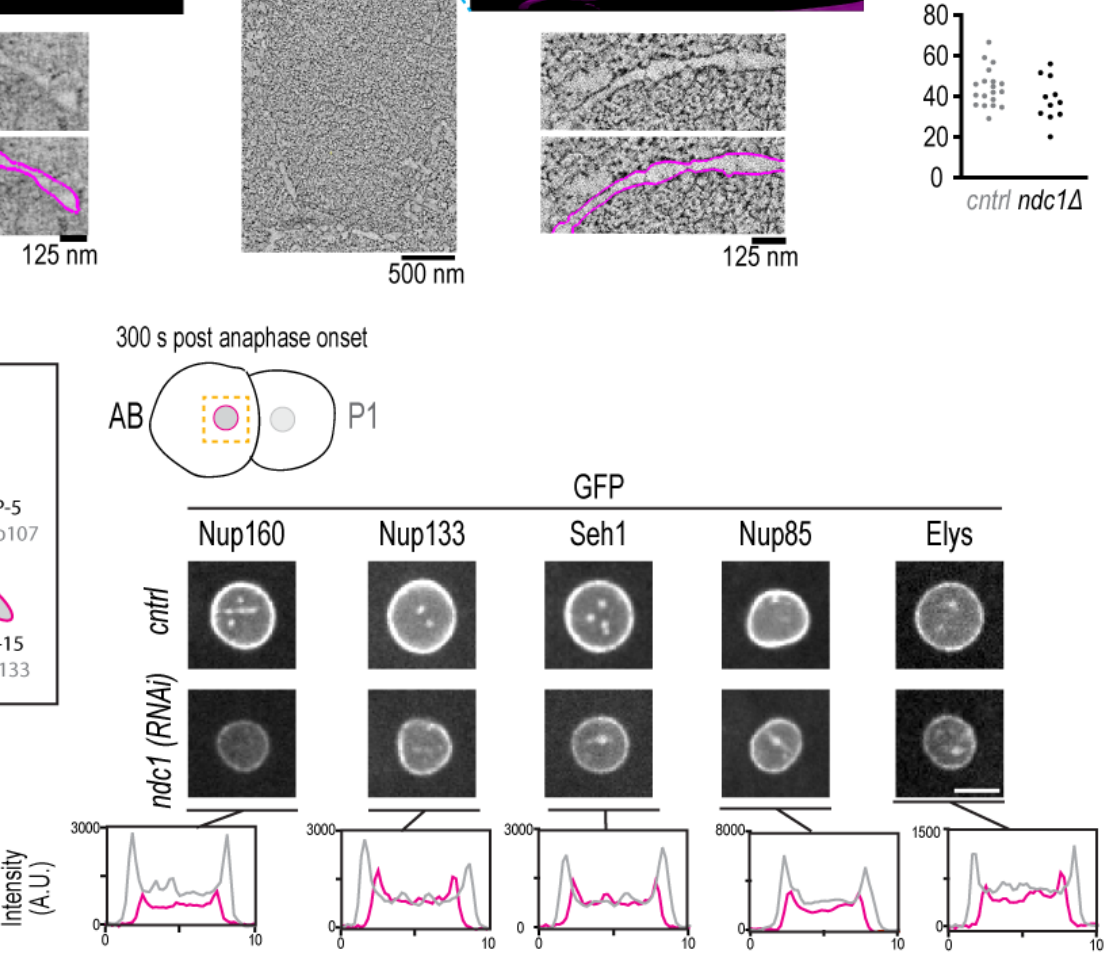

E

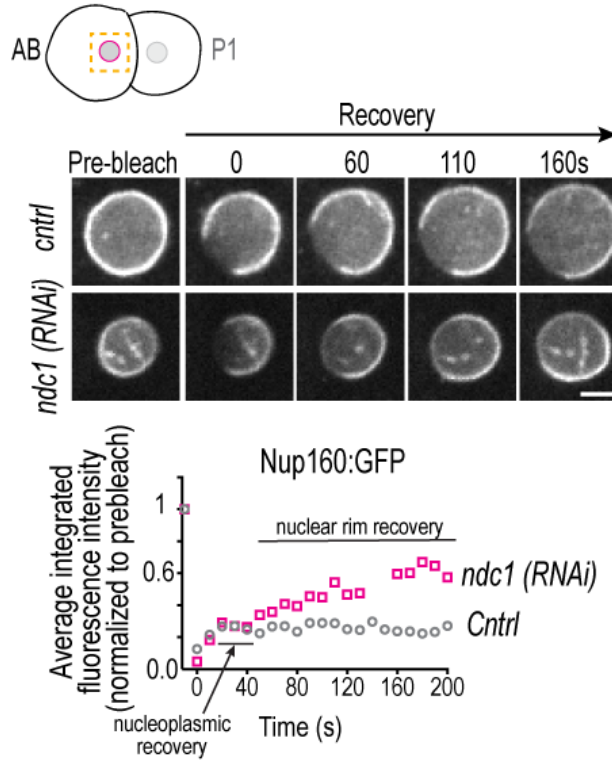

$\mathbf{F}$

NDC1 ${ }^{\text {en:mNG }}$
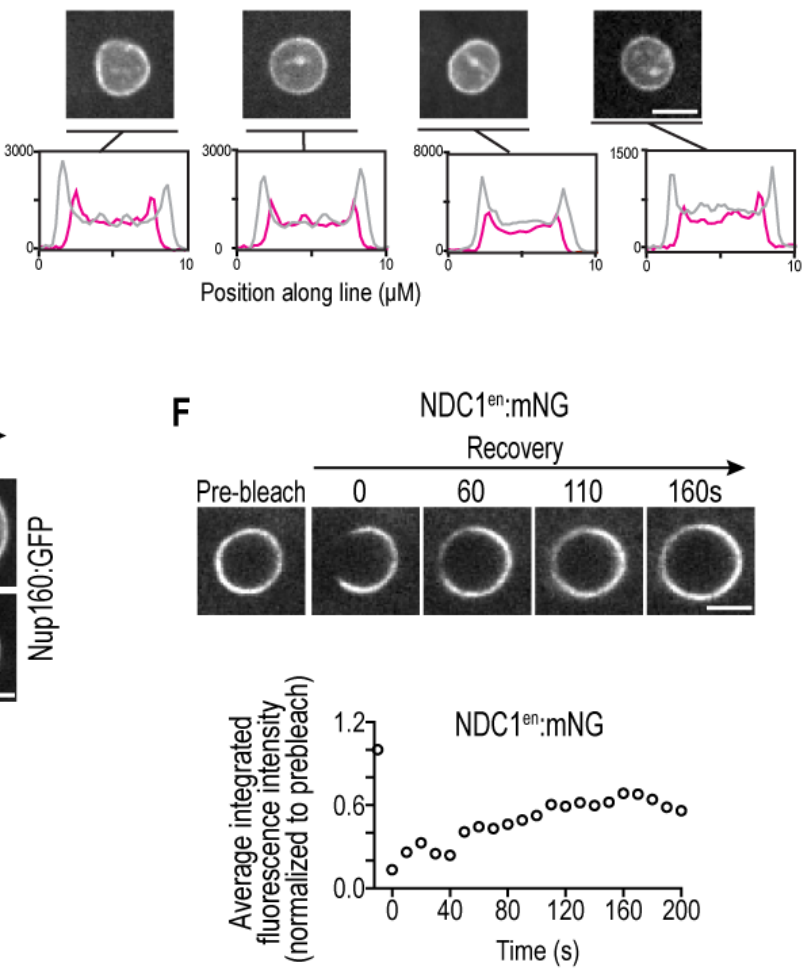
Figure 3. Ndc1 is mobile in the nuclear envelope and loss of Ndc1 results in fewer NPCs assembled on nascent NEs and a higher mobile pool of Nup160:GFP. (A, B) Overview images: z-slice from electron tomogram of nuclear formation timed relative to initiation of furrow ingression. 3D model: traced membranes (magenta) and NE holes $<100 \mathrm{~nm}$ (blue) for region shown as single z-slice in overview image. Magnified representative $z$ slice traced and untraced from electron tomogram is shown. (C) Plot of diameters of NE holes $<100 \mathrm{~nm}$. (D) Schematic of Y-complex with vertebrate (grey) and C. elegans (black) names shown. Magnified images of $A B$ nucleus from confocal series for each indicated marker (above) and line scans measuring background-corrected fluorescent intensities (below) for each condition. (E) Confocal images from time lapse series of fluorescence recovery after photobleaching of Nup160:GFP for indicated conditions is shown (above). Plot of average fluorescence intensity of bleached region over time normalized to the prebleach intensity for each condition is shown (below). (F) Confocal images from time lapse series of fluorescence recovery after photobleaching of $\mathrm{Ndc}^{\text {en:GFP }}{ }^{\text {is shown }}$ (above). Plot of average fluorescence intensities of bleached region over time normalized to the prebleach intensity for each condition is shown (below). Scale bars, $5 \mu \mathrm{m}$. 
Mauro Figure 4
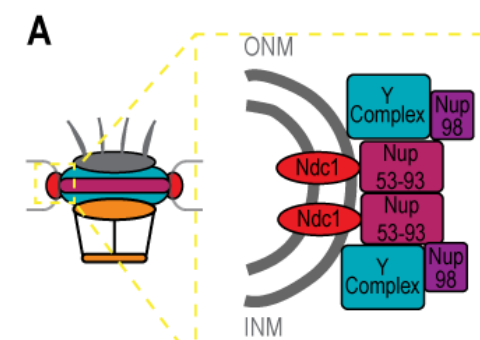

B

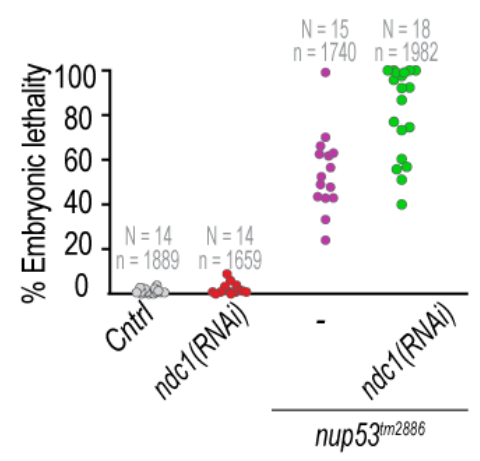

C -100 s relative to $P C$ regression

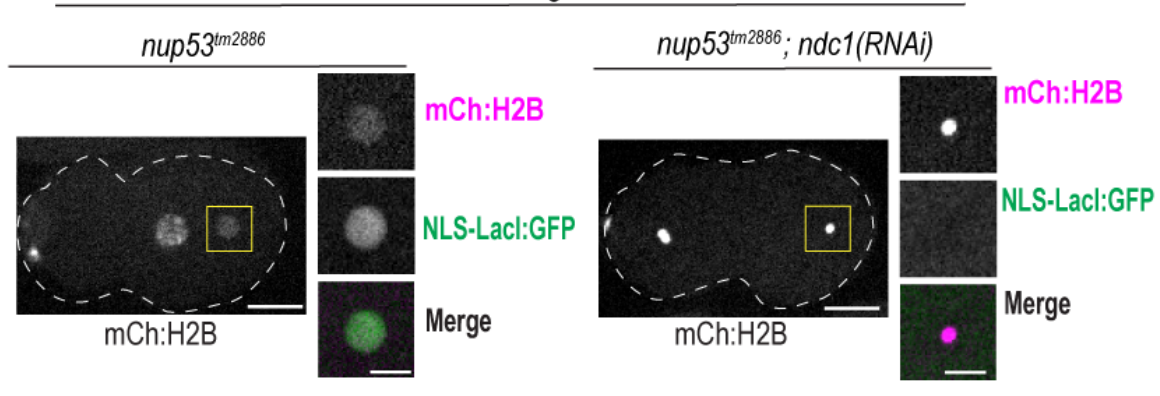

D -100 s relative to $P C$ regression Nup160:GFP
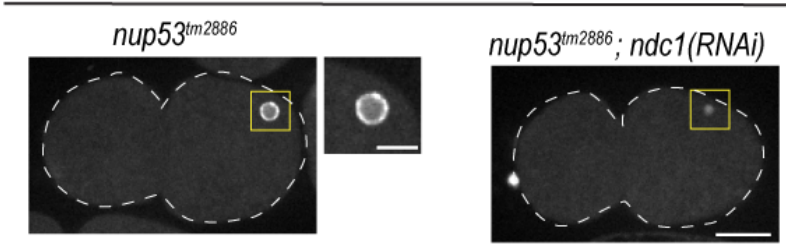

$\mathbf{F}$

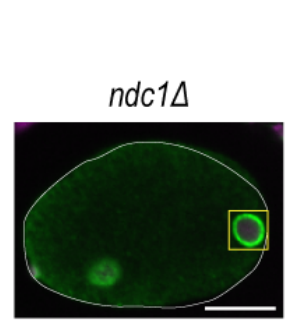

anti-mAb414 DAPI
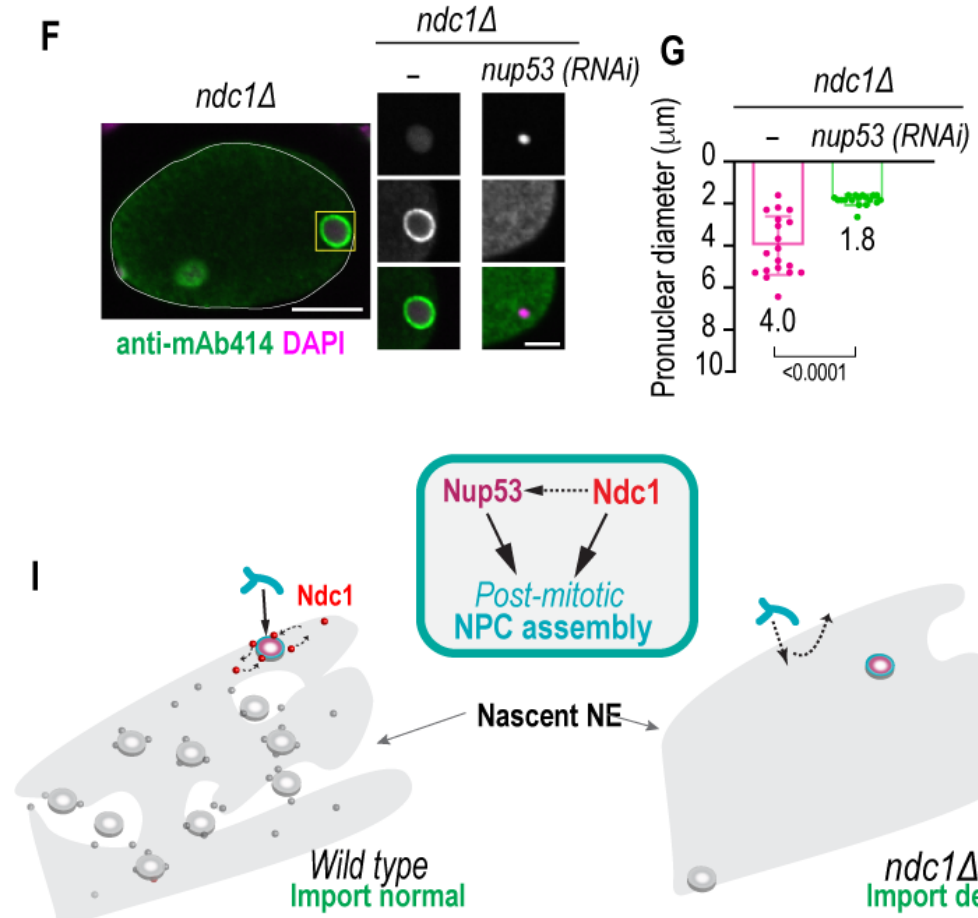

Wild type
mport norm
E

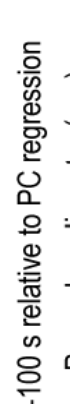

(1)

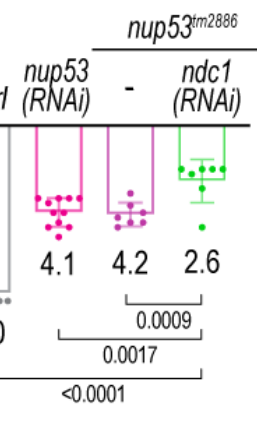

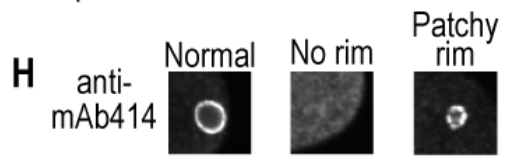

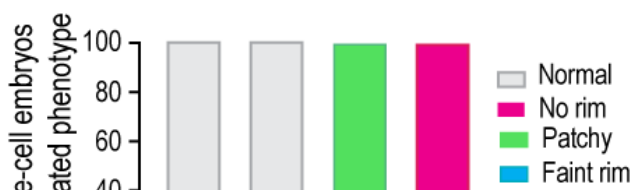

$n d c 1 \Delta$

Import delayed 
Figure 4. Ndc1 coordinates with Nup53 in nuclear assembly. (A) Schematic of NPC (left) and NPC subcomplex organization (right) is shown. (B) Plot of percentage embryonic lethality for indicated conditions. $\mathrm{N}=$ number of worms. $\mathrm{n}=$ number of embryos. (C) Confocal overview and magnified images of embryo from a time lapse series of indicated markers for indicated conditions. (D) Confocal overview and magnified images of embryo from a time lapse series of Nup160:GFP for indicated conditions. (E) Pronuclear diameter for indicated conditions at indicated time point. $\mathrm{n}=$ number of embryos. cntrl $\mathrm{n}=9$, nup53(RNAi) $\mathrm{n}=11$, nup53 ${ }^{\text {tm2886 }} \mathrm{n}=7$,

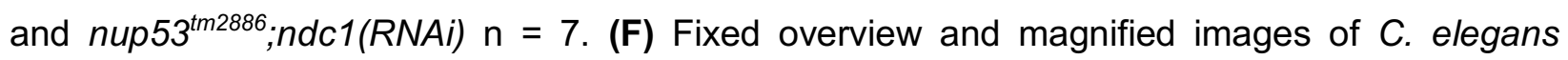
embryos immunostained for mAb414 and DAPI in indicated conditions. Scale bars, $10 \mu \mathrm{m}$ for overview image and $5 \mu \mathrm{m}$ for magnified images. (G) Pronuclear diameter for indicated conditions at indicated time point. $\mathrm{n}=$ number of embryos. $n d c 1 \Delta \mathrm{n}=20$ and $n d c 1 \Delta ; n u p 53(R N A i) \mathrm{n}=18 .(\mathbf{H})$ Magnified images of paternal pronucleus from fixed one-cell stage embryos immunostained with mAb414 for indicated conditions (top). Plot of mAb414 appearance surrounding chromatin under indicated conditions (bottom). A two-way ANOVA was used to determine statistical significance between indicated conditions. $\mathrm{n}=$ number of embryos. Scale bars, $10 \mu \mathrm{m}$ for overview image and $5 \mu \mathrm{m}$ for magnified images. (I) Schematic representation of NE formation with and without Ndc1. Schematic of a simplified genetic pathway highlighting the parallel contributions of Ndc1 and Nup53 to nuclear assembly. 
A Generation of $n d c 1 \Delta$ by NHEJ

ndc1 genomic locus

$250 \mathrm{bp}$

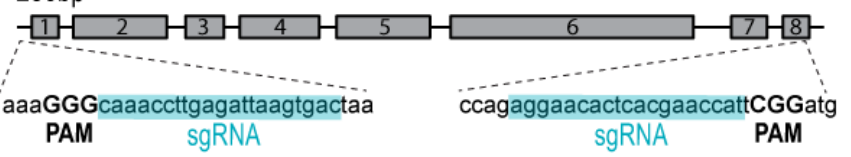

5 ' guide (37bp upstream of ATG) $\quad 3$ ' guide (24bp upstream of stop codon)
B

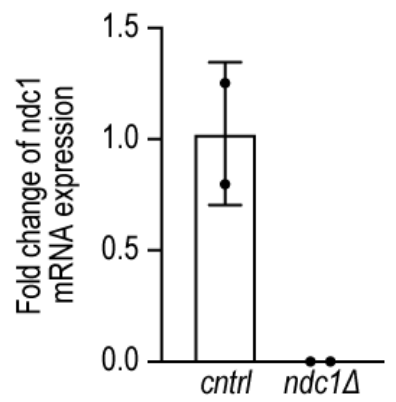

C

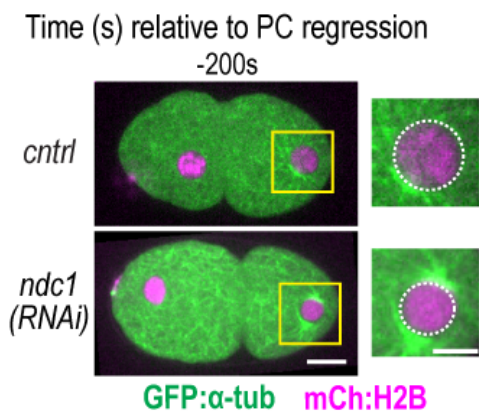

D ndc1 genomic locus

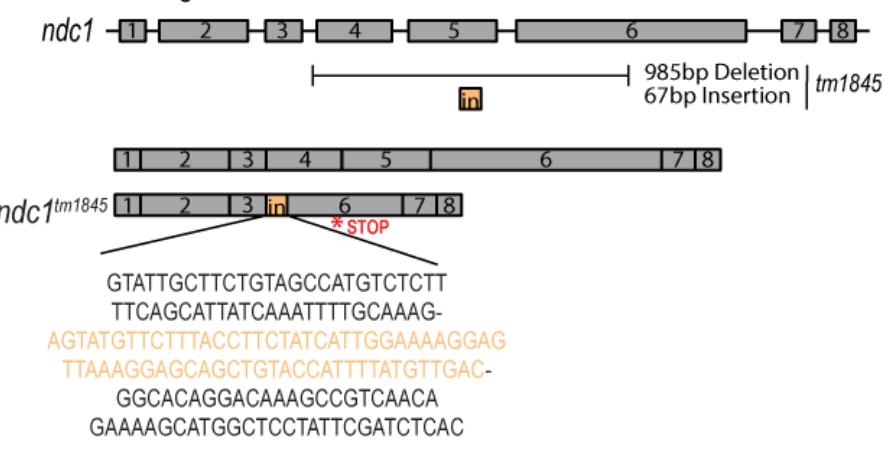

$F$

E

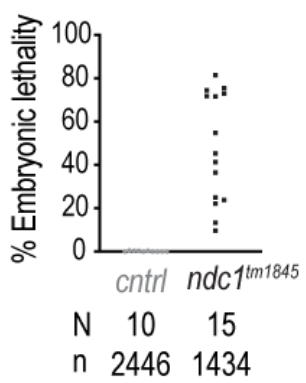

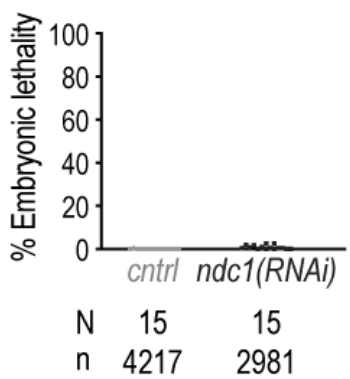

Figure S1. Generation and characterization of mutant ndc1 alleles and RNAi-depletion of ndc1. Related to Figure 1.

(A) Schematic of CRISPR guides to generate ndc1 null allele. (B) Plot of $n d c 1$ mRNA fold change for control and ndc1 $\Delta$ worms. Mean +/-SD. (C) Confocal overview and magnified images of onecell stage embryo from a time lapse series of indicated markers for indicated conditions. Scale bars, $10 \mu \mathrm{m}$ for overview image and $5 \mu \mathrm{m}$ for magnified images. (D) Schematic of $n d c 1$ genomic locus (top) and tm1845 allele (below). (E-F) Plots of percentage embryonic lethality for indicated conditions. $\mathrm{N}=$ number of worms. $\mathrm{n}=$ number of embryos. 
A

A

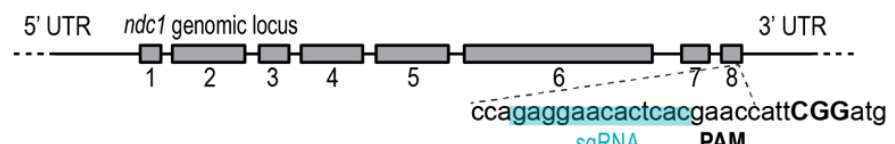

sgRNA PAM

Ndc1 $1^{\text {en }}: m N G$

3 ' guide (24bp upstream of stop codon)

CRISPR-Cas9 gene editting of $n d c 1$ genomic locus Stop Codon

(..-口

Ndc1 $1^{\text {en }}:$ mRuby

CRISPR-Cas9 gene editting of $n d c 1$ genomic locus

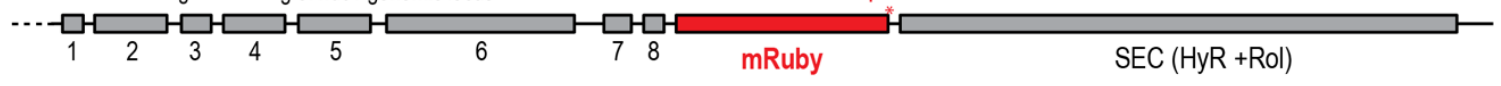

\section{B}

Ndc1en:mNG
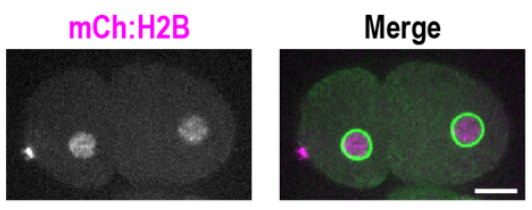

C Time (s) relative to $\mathrm{PC}$ regression

$-300 \mathrm{~s}$

$300 \mathrm{~s}$

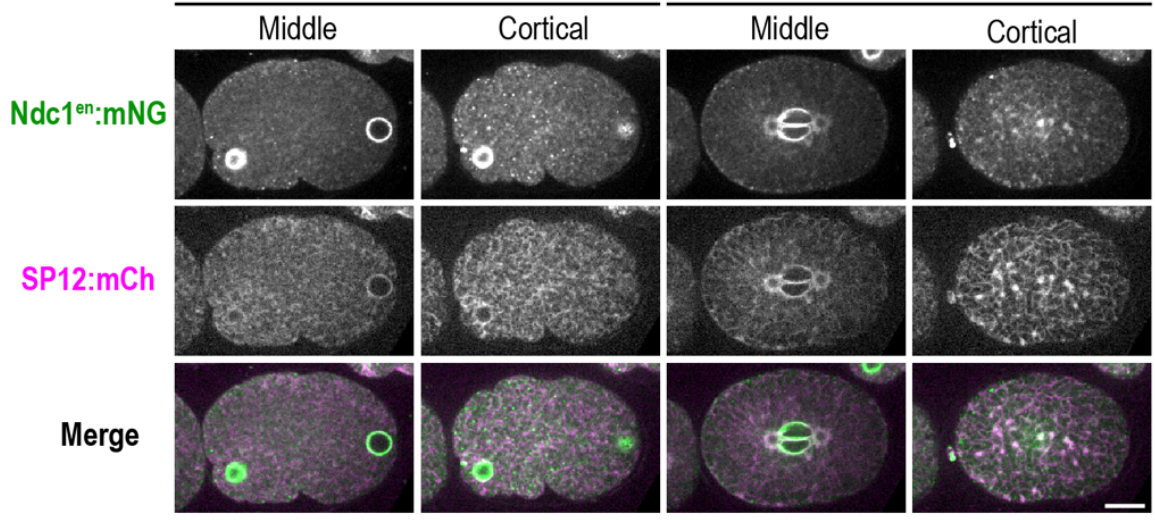

Time (s) relative to furrow ingression

D

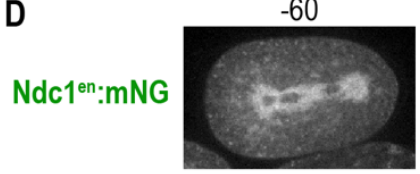

$\mathrm{SP} 12: \mathrm{mCh}$

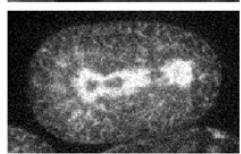

Merge

E

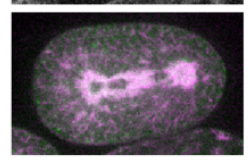

$-40$
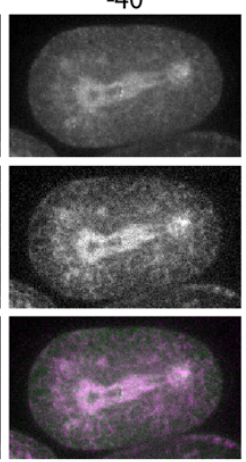
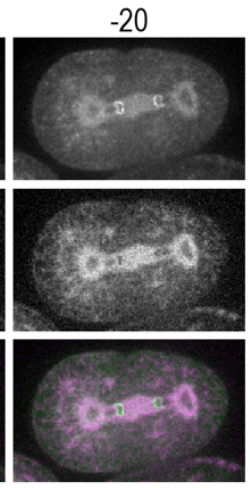
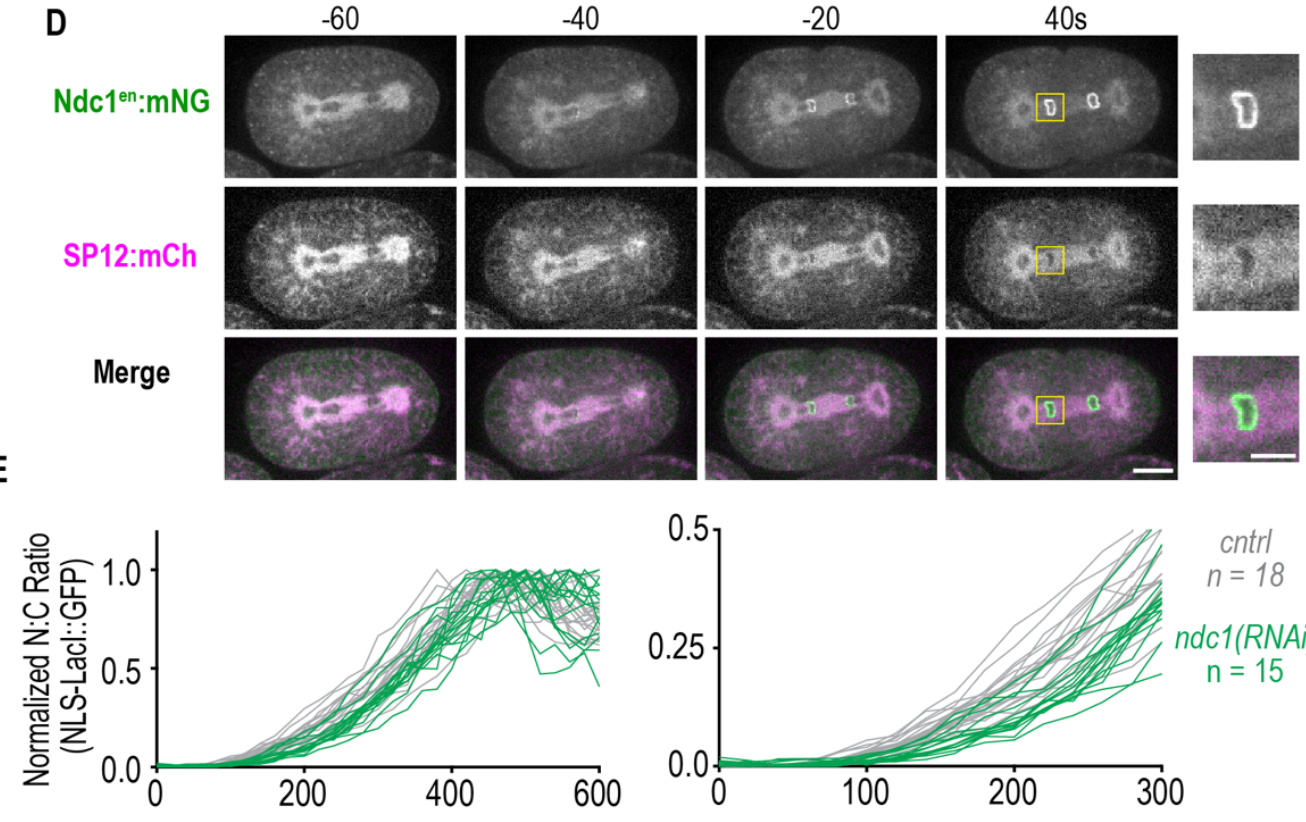

cntrl

$n=18$

$n d c 1$ (RNAi)

$\mathrm{n}=15$

Time (s) relative to anaphase onset 
Figure S2. Ndc1 ${ }^{\text {en }}: \mathrm{mNG}$ is recruited early to the nuclear rim and localizes to the ER and cytoplasmic puncta and nuclear transport is delayed in post-mitotic nuclei without Ndc1. Related to Figure 2.

(A) Schematic of ndc1 genomic locus and CRISPR guide to generate $n d c 1^{\text {en: }}: m N G$ and

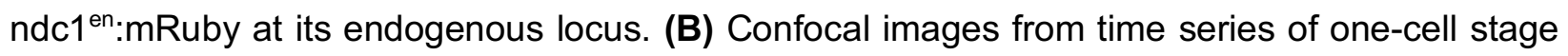
embryo expressing $\mathrm{Ndc}^{\mathrm{en}}: \mathrm{mNG}$ and $\mathrm{mCh}: \mathrm{H} 2 \mathrm{~B}$. (C) Confocal images from time series of one-cell stage embryo expressing Ndc $1^{\text {en }}: \mathrm{mNG}$ and SP12:GFP to mark the ER. (D) Confocal images from time series of two-cell stage embryo expressing $\mathrm{Ndc} 1^{\mathrm{en}}: \mathrm{mNG}$ and SP12:mCh during mitotic nuclear formation. Scale bars, $10 \mu \mathrm{m}$ and $5 \mu \mathrm{m}$ for magnified images. (E) Plot of normalized nuclear to cytoplasmic ratio of GFP:NLS-Lacl for indicated conditions. $n=$ number of embryos. 


\section{Mauro Figure S3}

A

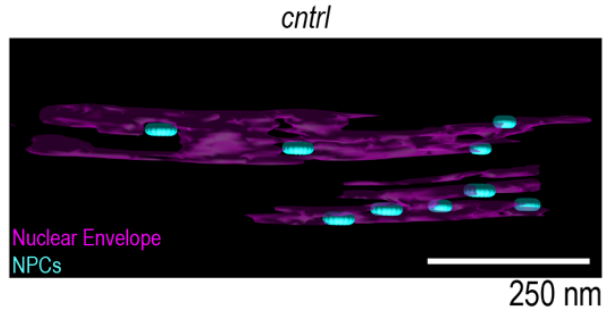

B

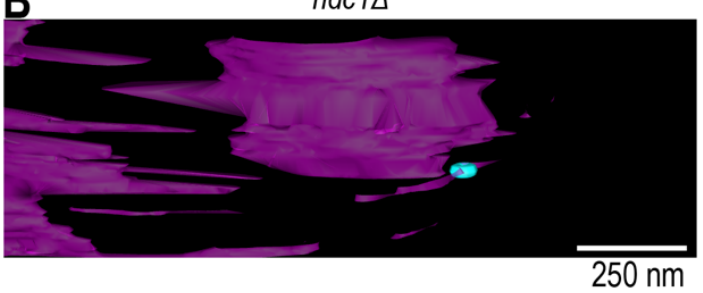

Time (s) relative to anaphase onset
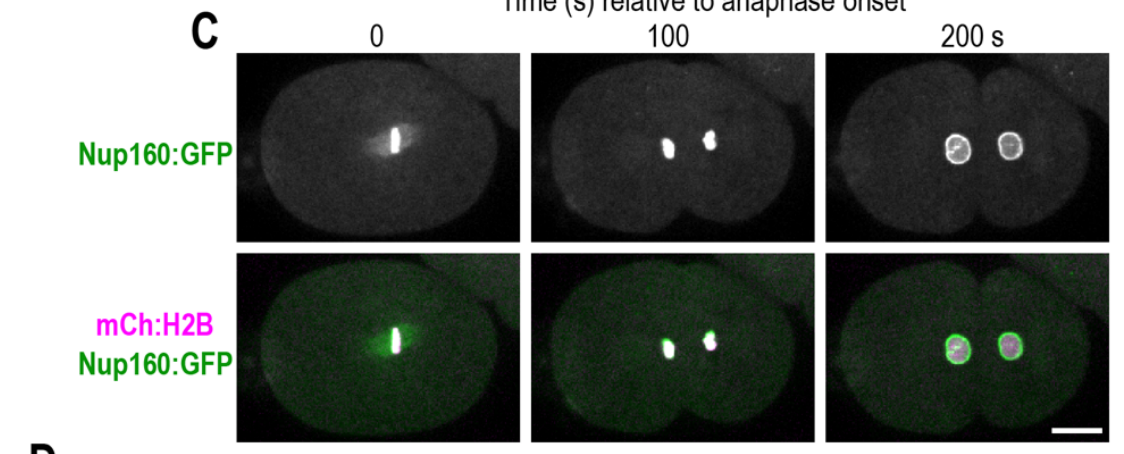
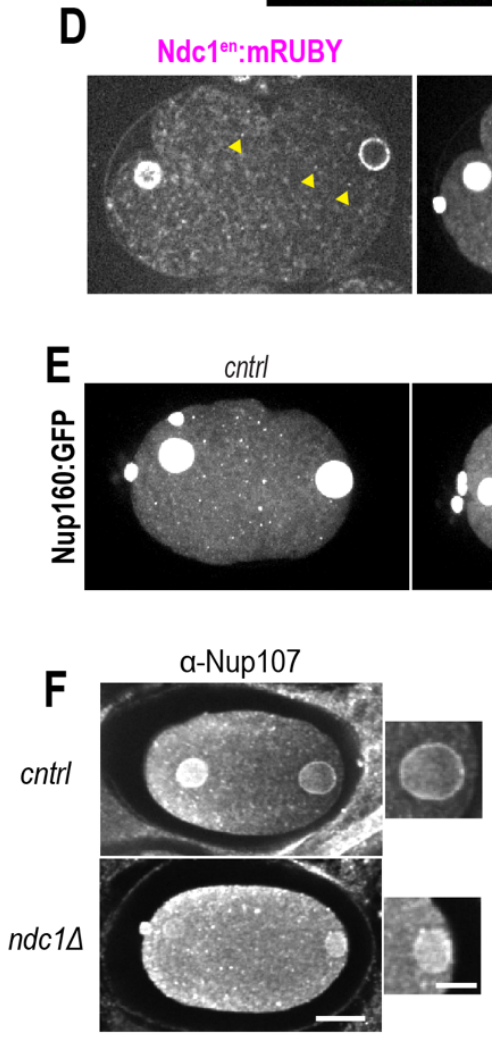

H cntrl ndc1 100kd50kd- — a-Tubulin
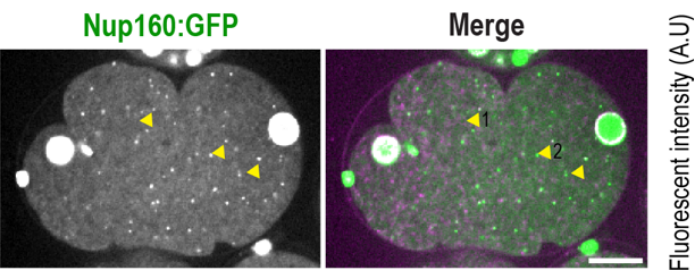

Average number of cytoplasmic $\operatorname{ndc1}(R N A i)$

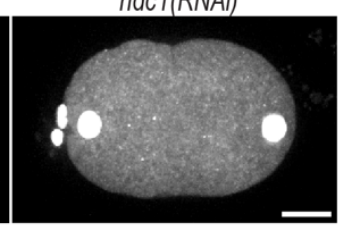

Nup160::GFP puncta

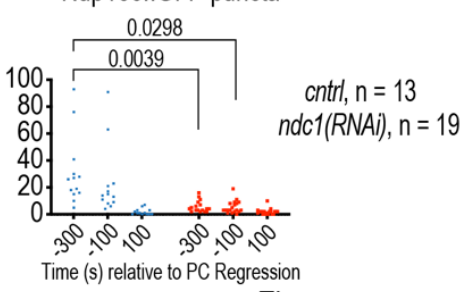

NE:Cytoplasmic Ratio
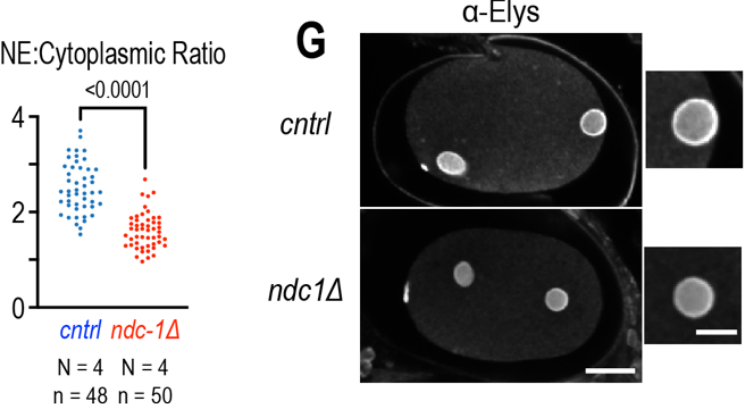

NE:Nucleoplasmic Ratio

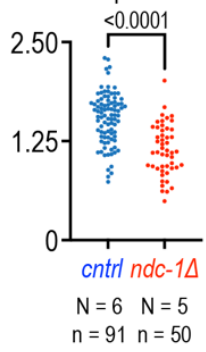


Figure S3. Lower nuclear pore density and levels of outer ring scaffold nucleoporins at the nuclear rim and cytoplasmic puncta resulting from loss of Ndc1. Related to Figure 3.

(A, B) 3D model of tomograms of NE reformation showing membranes (magenta) and NE holes $<100 \mathrm{~nm}$ (blue) for indicated conditions. (C) Confocal images from time series of mitotic nuclear formation relative to anaphase onset with indicated markers in control embryos. (D) Confocal overview and magnified images of embryo from a time lapse series of Nup160:GFP and

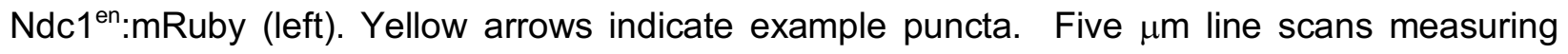
background-corrected fluorescent intensities for each marker drawn across two representative cytoplasmic puncta with co-localized Ndc1 and Nup160 signal (right). Scale bars, $10 \mu \mathrm{m}$ and 2.5 $\mu \mathrm{m}$ for magnified images. (E) Max projection confocal images of Nup160:GFP for indicated conditions (left). Plot of average number of cytoplasmic Nup160:GFP structures under indicated conditions at time points relative to PC regression (right). Scale bar, $10 \mu \mathrm{m}$. Students T-test was used to determine statistical significance. (F) Fixed overview and magnified images of $C$. elegans embryos immunostained with antibodies that recognize Nup107 for indicated conditions (left). Plot of NE to cytoplasmic ratio for normalized Nup107 fluorescence intensity (right). Scale bars, 10 $\mu \mathrm{m}$ and $5 \mu \mathrm{m}$ for magnified images. (G) Fixed overview and magnified images of $C$. elegans embryos immunostained for antibodies against Elys ${ }^{\text {Mel-28 }}$ for indicated conditions (right). Plot of $\mathrm{NE}$ to nucleoplasmic ratio for normalized Elys ${ }^{\mathrm{Mel}-28}$ fluorescence intensity (right). Scale bars, 10 $\mu \mathrm{m}$ and $5 \mu \mathrm{m}$ for magnified images. (H) Immunoblot of whole worm lysates probed for antibodies that recognize Nup107 and $\alpha$-tubulin for indicated conditions. 


\section{Mauro Figure S4}
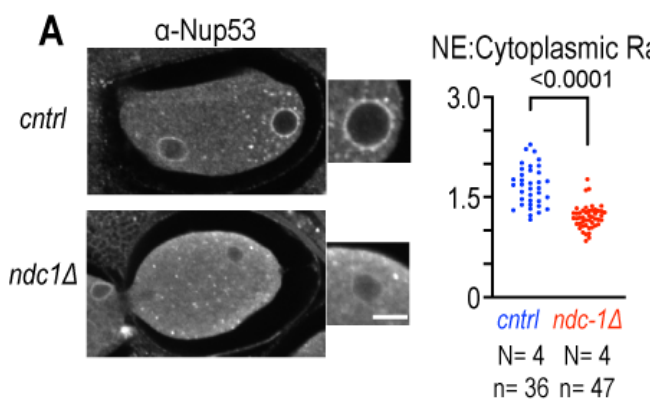

\section{C}
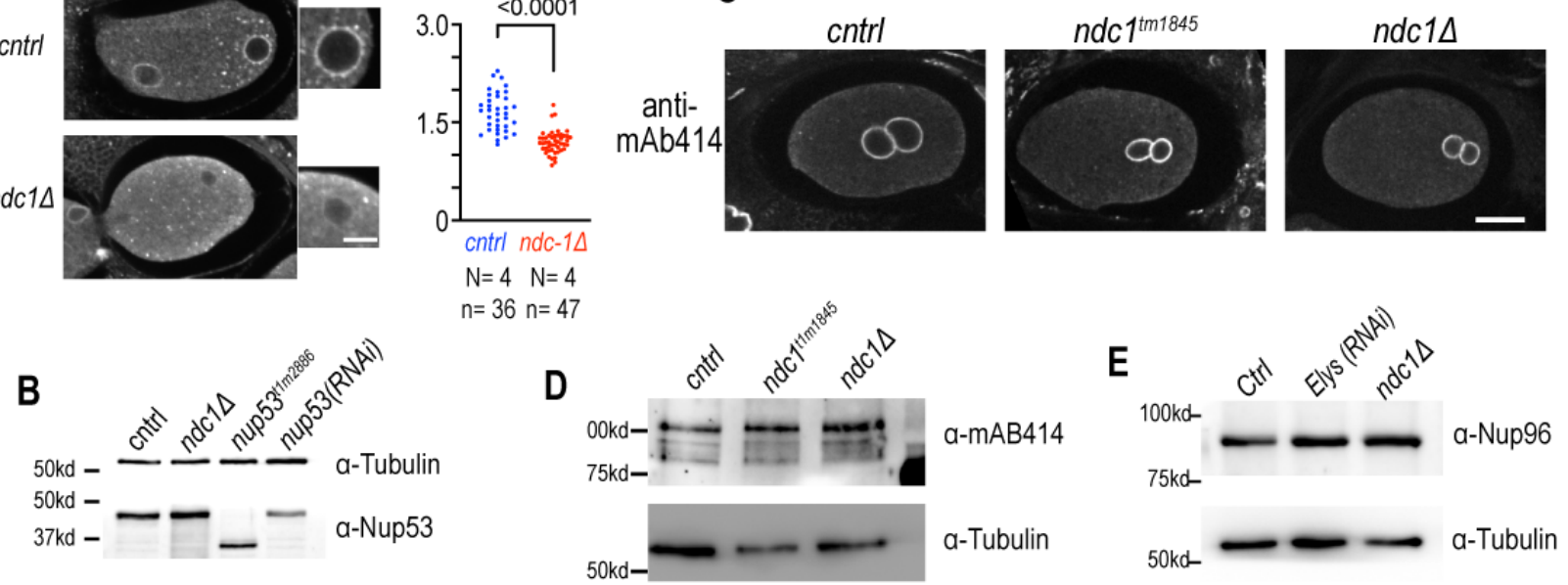

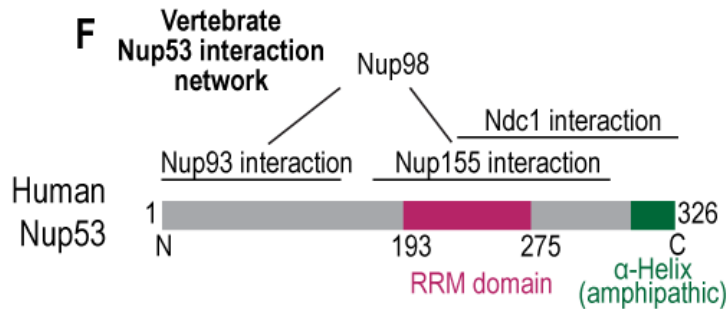

RRM= RNA recognition motif (Nup53 dimerization)

Binding sites based on Human nup53

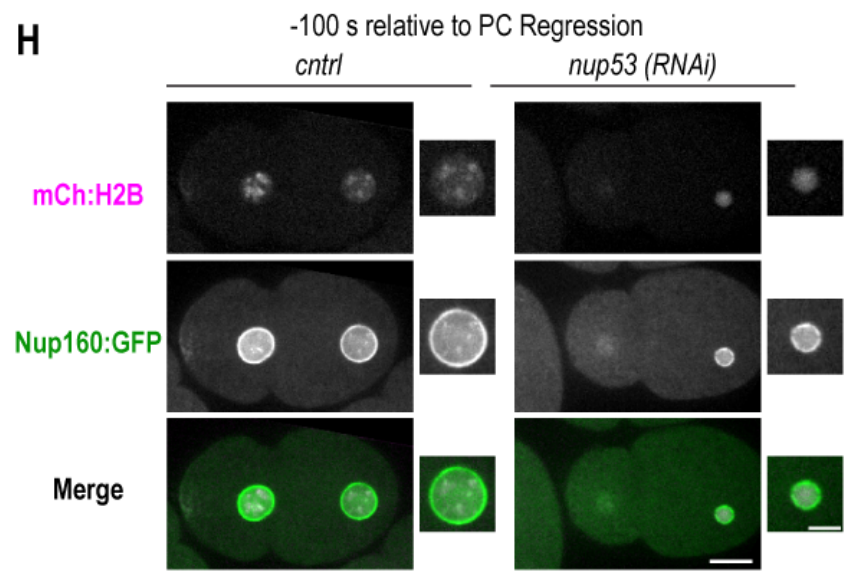

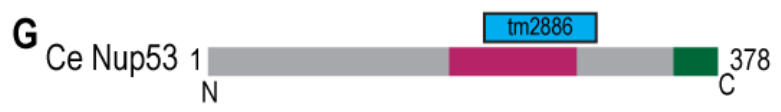

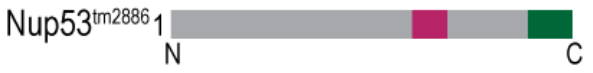

I
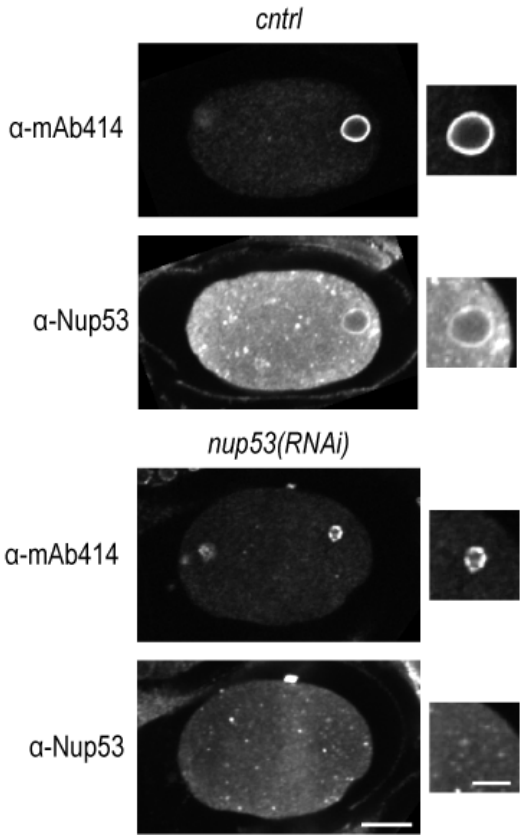
Figure S4. Characterization of levels and localization of nucleoporins in ndc1 mutant and nup53 mutant alleles. Related to Figure 4.

(A) Fixed overview and magnified images of C. elegans embryos immunostained for antibodies that recognize Nup53 for indicated conditions (left). Plot of NE to cytoplasmic ratio for normalized Nup53 fluorescence intensity (right). (B) Immunoblot of whole worm lysates probed for antibodies that recognize Nup53 and $\alpha$-tubulin for indicated conditions. (C) Fixed overview images of $C$. elegans oocytes immunostained for mAb414 for indicated conditions. Scale bars, $10 \mu \mathrm{m}$. (D) Immunoblot of whole worm lysates probed for mAb414 and a-tubulin for indicated conditions. (E) Immunoblot of whole worm lysates probed for Nup96 and a-tubulin for indicated conditions. (F) Schematic of Nup53 domain architecture and binding interactions based on human Nup53 binding sites. (G) Schematic of $C$ elegans Nup53 domain architecture and the resulting mutant protein resulting from the nup $53^{\operatorname{tm} 2886}$ allele. $(\mathbf{H})$ Confocal overview and magnified images of one-cell stage embryo from a time lapse series expressing Nup160:GFP and mCh:H2B for indicated conditions. Scale bars, $10 \mu \mathrm{m}$ and $5 \mu \mathrm{m}$ for magnified images. (I) Fixed overview and magnified images of $C$. elegans embryos immunostained for mAb414 and Nup53 for indicated conditions. Scale bars, $10 \mu \mathrm{m}$ and $5 \mu \mathrm{m}$ for magnified images. 


\section{Key Resources}

\begin{tabular}{|c|c|c|}
\hline REAGENT or RESOURCE & SOURCE & IDENTIFIER \\
\hline \multicolumn{3}{|l|}{ Antibodies } \\
\hline mouse $\alpha$-alpha-tubulin & Millipore Sigma & $\begin{array}{l}\text { Cat\#05-829; RRID: } \\
\text { AB_310035 }\end{array}$ \\
\hline mouse mAb414 & Biolegend & $\begin{array}{l}\text { Cat\# 902907; } \\
\text { RRID: } \\
\text { AB_2734672 }\end{array}$ \\
\hline rabbit $\alpha-N P P-5 / N u p 107$ & [19] & $\mathrm{N} / \mathrm{A}$ \\
\hline rabbit a-NPP-10N/Nup98 & [19] & $\mathrm{N} / \mathrm{A}$ \\
\hline rabbit $\alpha-N P P-10 C / N u p 96$ & [19] & $\mathrm{N} / \mathrm{A}$ \\
\hline rabbit a-NPP-19/Nup53 & [19] & $\mathrm{N} / \mathrm{A}$ \\
\hline rabbit $\alpha-M E L-28 / E l y s$ & [19] & $\mathrm{N} / \mathrm{A}$ \\
\hline Rhodamine RedX Donkey a rabbit IgG & Jackson Immuno & $\begin{array}{l}\text { Cat\#711-295-152 } \\
\text {; RRID: } \\
\text { AB 2340613 }\end{array}$ \\
\hline FITC Goat $\alpha$ mouse lgG & Jackson Immuno & $\begin{array}{l}\text { Cat\#115-095-146; } \\
\text { RRID: } \\
\text { AB 2338599 }\end{array}$ \\
\hline Goat a mouse IgG-HRP & Thermo Fisher & $\begin{array}{l}\text { Cat\#31430; RRID: } \\
\text { AB 228307 }\end{array}$ \\
\hline Goat $\alpha$ rabbit IgG-HRP & Thermo Fisher & $\begin{array}{l}\text { Cat\#31460; RRID: } \\
\text { AB 228341 }\end{array}$ \\
\hline \multicolumn{3}{|l|}{ Chemicals, peptides, and recombinant proteins } \\
\hline Clarity Max Western ECL Substrate & BIO-Rad & $1705060 S$ \\
\hline \multicolumn{3}{|l|}{ Critical commercial assays } \\
\hline MEGAscript T3 Transcription Kit & Invitrogen & Cat\# AM1338 \\
\hline MEGAscript T7 Transcription Kit & Invitrogen & Cat\# AM1334 \\
\hline \multicolumn{3}{|l|}{ Experimental models: Organisms/strains } \\
\hline C. elegans: Strain N2: wild-type (ancestral) & $\begin{array}{l}\text { Caenorhabditis } \\
\text { Genetics Center }\end{array}$ & $\mathrm{N} 2$ \\
\hline $\begin{array}{l}\text { C. elegans: Strain OD997: unc-119(ed3)III; } \\
\text { ItSi231[pNH16; Pmel-28::GFP-mel-28; cb-unc- } \\
\text { 119(+)]ll;; ; Itls37[pAA64; pie-1/mCherry::his-58; } \\
\text { unc-119(+)] IV }\end{array}$ & {$[25]$} & OD997 \\
\hline $\begin{array}{l}\text { C. elegans: Strain OD999: unc-119(ed3)III; } \\
\text { ItSi245[pNH42; Pnpp-18::GFP-npp-18; cb-unc- } \\
\text { 119(+)]II; It/s37[pAA64; pie-1/mCherry::his-58; unc- } \\
119(+)] \text { IV }\end{array}$ & [25] & OD999 \\
\hline $\begin{array}{l}\text { C. elegans: Strain OD1496: unc-119(ed3)III; } \\
\text { ItSi464[pNH103; Pmex-5::npp-6::GFP::tbb-2 } \\
\text { 3'UTR; cbunc-119(+)]l; ItIs37[pAA64; pie- } \\
\text { 1/mCherry::his-58; unc- } 119 \text { (+)] IV }\end{array}$ & [25] & OD1496 \\
\hline $\begin{array}{l}\text { C. elegans: Strain OD1498: unc-119(ed3)III; } \\
\text { ItSi465[pNH104; Pmex-5::npp-15::GFP::tbb-2 } \\
\text { 3'UTR; cb-unc-119(+)]l; It/s37[pAA64; pie- } \\
\text { 1/mCherry::his-58; unc-119 (+)] IV }\end{array}$ & [25] & OD1498 \\
\hline
\end{tabular}




\begin{tabular}{|c|c|c|}
\hline $\begin{array}{l}\text { C. elegans: Strain OD1499: unc-119(ed3)III; } \\
\text { ItSi463[pNH102; Pmex-5::npp2::GFP::tbb-2 3'UTR; } \\
\text { cb- unc-119(+)]l; It/s37[pAA64; pie-1/mCherry::his- } \\
\text { 58; unc-119(+)] IV }\end{array}$ & [25] & OD1499 \\
\hline $\begin{array}{l}\text { C. elegans: Strain OD2400: ItSi896[pNH152; } \\
\text { Pgsp-2::GSP-2::GFP; cb-unc-119(+)]l; } \\
\text { ItIs37[pAA64; pie-1/mCherry::his-58; unc-119 (+)] } \\
\text { IV }\end{array}$ & [25] & OD2400 \\
\hline $\begin{array}{l}\text { C. elegans: Strain SBW32: unc-119(ed3) III; It/s24 } \\
\text { [pAZ132; pie-1/GFP::tba-2; unc-119 (+)]; It/s37 } \\
\text { [(pAA64) pie-1p::mCherry::his-58 + unc-119(+)] IV }\end{array}$ & [34] & SBW32 \\
\hline $\begin{array}{l}\text { C. elegans: Strain SBW47:unc-119(ed3) III; ItIs37 } \\
\text { [pie-1/mCherry::his-58; unc-119 (+)] IV; It/s75 } \\
\text { [Ppie-1/GFP::TEV-Stag::Lacl + unc-119(+)]. }\end{array}$ & [16] & SBW47 \\
\hline $\begin{array}{l}\text { C. elegans: Strain SBW56: } n p p-22(t m 1845 / n T 1) V \\
7 x \text { outcrossed }\end{array}$ & This study & SBW56 \\
\hline $\begin{array}{l}\text { C. elegans: Strain SBW83: npp-22 (sbw4) V, } 4 x \\
\text { outcrossed }\end{array}$ & This study & SBW83 \\
\hline $\begin{array}{l}\text { C. elegans: Strain SBW84: unc-119(ed3) III; } \\
\text { bqSi242 [lem-2p::lem-2::mCherry + unc-119(+)] IV; } \\
\text { ItIs75 [(pSK5) pie-1::GFP::TEV-STag::Lacl + unc- } \\
119(+)] .\end{array}$ & This study & SBW84 \\
\hline $\begin{array}{l}\text { C. elegans: Strain SBW191: npp-19(tm2886) } 6 x \\
\text { outcrossed }\end{array}$ & This study & SBW191 \\
\hline $\begin{array}{l}\text { C. elegans: Strain SBW244: ndc1::mNeonGreen } \\
\text { (sbw8)-4x outcrossed }\end{array}$ & This study & SBW244 \\
\hline $\begin{array}{l}\text { C. elegans: Strain SBW252: unc-119(ed3) III; It/s } \\
76 \text { [pAA178; pie-1/mCherry:SP-12; unc-119 (+)]; } \\
\text { ndc1::mNEON (sbw8)-4x outcrossed }\end{array}$ & This study & SBW252 \\
\hline $\begin{array}{l}\text { C. elegans: Strain SBW254:bqSi242 [lem-2p::lem- } \\
2:: m \text { Cherry + unc-119(+)] IV.; ndc1::mNeonGreen } \\
\text { (sbw8)-4x outcrossed }\end{array}$ & This study & SBW254 \\
\hline $\begin{array}{l}\text { C. elegans: Strain SBW260: npp-19(tm2886) } \\
\text { outcross 6x; (unc-119(ed3)III; ItSi464[pNH103; } \\
\text { Pmex-5::npp6::GFP::tbb-2 3'UTR; cb-unc-119(+)]I) }\end{array}$ & This Study & SBW260 \\
\hline $\begin{array}{l}\text { C. elegans: Strain SBW266: npp-19(tm2886) } \\
\text { outcross 6x; unc-119(ed3) III; Itls37 [pAA64; pie- } \\
\text { 1/mCHERRY::his-58; unc-119 (+)] IV; Itls75 } \\
\text { [(pSK5) pie-1::GFP::TEV-STag::Lacl + unc-119(+)]. }\end{array}$ & This Study & SBW266 \\
\hline $\begin{array}{l}\text { C. elegans: Strain SBW293: (unc-119(ed3)III; } \\
\text { ItSi464[pNH103; Pmex-5::npp6::GFP::tbb-2 } \\
\text { 3'UTR; cb-unc-119(+)]l) V; ndc1::mRuby (sbw14)- } \\
\text { 4x outcrossed }\end{array}$ & This study & SBW293 \\
\hline \multicolumn{3}{|l|}{ Oligonucleotides } \\
\hline
\end{tabular}




\begin{tabular}{|c|c|c|}
\hline $\begin{array}{l}\text { T3 primer for dsRNA targeting } n p p-22 \text { (ndc1) } \\
\text { Forward: } \\
\text { AATTAACCCTCACTAAAGGCCCGCCTCCATATA } \\
\text { CAGTTC }\end{array}$ & This Study & $\mathrm{N} / \mathrm{A}$ \\
\hline $\begin{array}{l}\text { T7 primer for dsRNA targeting } n p p-22 \text { (ndc1) } \\
\text { Reverse: } \\
\text { TAATACGACTCACTATAGGTGTCAATGGCTGCA } \\
\text { ATGAGT }\end{array}$ & This Study & N/A \\
\hline $\begin{array}{l}\text { T3 primer for dsRNA targeting npp-7 (nup153) } \\
\text { Forward: } \\
\text { AATTAACCCTCACTAAAGGGTTCCTGCCACAAT } \\
\text { TCCAGT }\end{array}$ & This Study & N/A \\
\hline $\begin{array}{l}\text { T7 primer for dsRNA targeting npp-7 (nup153) } \\
\text { Reverse: } \\
\text { TAATACGACTCACTATAGGCTTGTAGACGATGC } \\
\text { AGCACC }\end{array}$ & This Study & N/A \\
\hline $\begin{array}{l}\text { T3 primer for dsRNA targeting npp-19 (nup53) } \\
\text { Forward: } \\
\text { AATTAACCCTCACTAAAGGCACCACCTCTTCGA } \\
\text { TCTCTTC }\end{array}$ & This Study & N/A \\
\hline $\begin{array}{l}\text { T7 primer for dsRNA targeting npp-19 (nup53) } \\
\text { Reverse: } \\
\text { TAATACGACTCACTATAGGTTTGTGCACTGAAC } \\
\text { GACTCC }\end{array}$ & This Study & N/A \\
\hline $\begin{array}{l}\text { npp-22/ndc1 (B0240.4) start of gene CRISPR } \\
\text { guide: AGTGAATTAGAGTTCCAAAC }\end{array}$ & This Study & N/A \\
\hline $\begin{array}{l}\text { npp-22/ndc1 (B0240.4) end of gene CRISPR } \\
\text { guide: AGGAACACTCACGAACCATT }\end{array}$ & This Study & N/A \\
\hline $\begin{array}{l}\text { npp-22/ndc1 qPCR primer forward: } \\
\text { AGCTGTTTCCTTGCCTTGTG }\end{array}$ & This Study & N/A \\
\hline $\begin{array}{l}\text { npp-22/ndc1 qPCR primer reverse: } \\
\text { TCTTGGCATCAGGAGAGCAT }\end{array}$ & This Study & N/A \\
\hline $\begin{array}{l}\text { hxk-2 (H25P06.1) qPCR primer forward: } \\
\text { tctgtgcgattttcttgtcg }\end{array}$ & [35] & N/A \\
\hline $\begin{array}{l}\text { hxk-2 (H25P06.1) qPCR primer reverse: } \\
\text { cgtgcaatcgcatcttctaa }\end{array}$ & [35] & N/A \\
\hline \multicolumn{3}{|l|}{ Recombinant DNA } \\
\hline Plasmid: PDD122, CRISPR-Cas9 & [36] & $\mathrm{N} / \mathrm{A}$ \\
\hline $\begin{array}{l}\text { Plasmid: pSB446; CRISPR npp-22/ndc1 guide } \\
\text { inserted using gibson-assembly) }\end{array}$ & This Study & N/A \\
\hline Plasmid: pBS-LL-mNG & [36] & $\mathrm{N} / \mathrm{A}$ \\
\hline $\begin{array}{l}\text { Plasmid: pSB448; } 1 \mathrm{~kb} \text { homology arms of } n p p- \\
\text { 22/ndc1 C-term inserted into pBS-LL-mNG using } \\
\text { gibson-assembly) }\end{array}$ & This Study & N/A \\
\hline Plasmid: LL-mRuby & This Study & N/A \\
\hline $\begin{array}{l}\text { Plasmid: pSB603; } 1 \mathrm{~kb} \text { homology arms of } n p p- \\
\text { 22/ndc1 C-term inserted into LL-mRuby using } \\
\text { gibson-assembly) }\end{array}$ & This Study & N/A \\
\hline
\end{tabular}




\begin{tabular}{|l|l|l|}
\hline FIJI (ImageJ) & NIH & $\begin{array}{l}\text { https://imagej.net/F } \\
\text { iji } \\
\text { RRID: } \\
\text { SCR_002285 }\end{array}$ \\
\hline IMOD Version 4.11 & $\begin{array}{l}\text { University of } \\
\text { Colorado } \\
\text { https://bio3d.colora } \\
\text { do.edu/imod/ }\end{array}$ \\
\hline GraphPad Prism 8/9 & GraphPad & N/A \\
\hline R Studio Version 1.2.5033 & R Studio, INC & $\begin{array}{l}\text { https://www.rstudio } \\
\text {.com }\end{array}$ \\
\hline
\end{tabular}

\section{RESOURCE AVAILABILITY}

\section{Lead Contact}

Further information and requests for reagents or resources should be direct to Shirin

Bahmanyar (shirin.bahmanyar@yale.edu).

\section{Materials availability}

C. elegans strains and plasmids from this study are available from the Lead Contact upon request.

\section{Data and code availability}

Raw data not included in this article are available from the corresponding author on request.

\section{EXPERIMENTAL MODEL AND SUBJECT DETAILS}

\section{Strain maintenance and generation}

The C. elegans strains used in this study are listed in the key resource table. Strains were maintained on nematode growth media (NGM) plates that were seeded with OP-50 Escherichia coli. Strains were grown at three possible temperatures: $15^{\circ} \mathrm{C}, 20^{\circ} \mathrm{C}$ and $25^{\circ} \mathrm{C}$.

\section{CRISPR-Cas9 deletion strain}

The deletion for ndc-1 (B0240.4) was generated using two CRISPR guides "crRNA", which were obtained using IDT's custom CRISPR guide algorithm (See Fig. S1A and key resource table for sequences). $1 \mu \mathrm{l}$ of the purified crRNAs were annealed to $1 \mu \mathrm{l}$ of trans-activating crRNA (tracrRNA) by incubating RNAs at 95 DC for two minutes in individual PCR tubes. A co-CRISPR crRNA was used for dpy-10. An injection mix with the following components and concentrations 
was set up and spun down for 30 minutes: at $4^{\circ} \mathrm{C}$; ndc-1 guide $1(11.7 \mu \mathrm{M})$, ndc-1guide 2 (11.7 $\mu \mathrm{M})$, purified Cas9 protein (qb3 Berkeley, $14.7 \mu \mathrm{M}), d p y-10$ guide $(3.7 \mu \mathrm{M})$ and a $d p y-10$ repair template $(29 \mathrm{ng} / \mathrm{mL}) .[37,38]$

The RNA-protein mix was then injected into the gonads of N2 adult worms, which were then allowed to recover for three days. After this recovery, F1 progeny were screened for a roller phenotype and 10-20 F1s were singled out to individual plates. These roller worms were allowed to produce progeny, which were then genotyped by PCR to detect the presence of the npp22/ndc1 deletion allele. The deletion strain was outcrossed four times to N2 (ancestral strain) worms before use and characterization.

CRISPR-Cas9 with SEC repair template for endogenous tagging

To endogenously tag ndc-1 with mNeonGreen and mRuby, a self-excising cassette (SEC) repair template approach was utilized. 950Bp homology arms from the five- and three prime-end of ndc1's stop codon were cloned into an SEC vector. This plasmid was co-injected with a Cas9 + ndc1 guide plasmid into the gonads of adult N2 worms. Worms were rescued to individual plates and allowed to recover for three days at $20^{\circ} \mathrm{C}$. Plates were screened for roller worms and positive plates were treated with approximately $200-300 \mu$ l of hygromycin $B(20 \mathrm{mg} / \mathrm{mL})$ and were allowed to recover for four-five days. Plates that had surviving rolling worms were screened for mNEONgreen or mRuby signal by microscopy. Finally, worms were outcrossed four times to N2 worms, before crossing to other fluorescent markers.

\section{METHOD DETAILS}

\section{RNAi}

Primers were designed to amplify a 200-1000bp region within a gene of interest, see key resource table for list of primers used. Primers were designed to amplify from within a single exon whenever possible. N2 gDNA or cDNA was used as a template for PCR, which in turn was purified and used in T3/T7 reverse transcription reactions (MEGAscript, Life Technologies). The synthesized RNAs were purified using phenol-chloroform and resuspended in 1X soaking buffer 
(32.7 mM Na2HPO4, 16.5 mM KH2PO4, $6.3 \mathrm{mM} \mathrm{NaCl,} 14.2 \mathrm{mM} \mathrm{NH} 4 \mathrm{Cl}$ ). RNA reactions were annealed at $68^{\circ} \mathrm{C}$ for 10 minutes followed by $37^{\circ} \mathrm{C}$ for 30 minutes. dsRNAs were brought to a final concentration of $\sim 2000 \mathrm{ng} / \mu \mathrm{l}$ whenever possible and $2-\mu$ l aliquots of the dsRNA were stored until use at $-80^{\circ} \mathrm{C}$. For each experiment, prior to injection, a fresh aliquot was diluted to $\sim 1000 \mathrm{ng} / \mu \mathrm{l}$ and centrifuged at $13,000 \mathrm{rpm}$ for 30 minutes at $4^{\circ} \mathrm{C} .0 .35 \mu$ of the diluted dsRNA was loaded into the back of hand pulled capillary needles and injected into the gut of L4 worms. Worms were rescued to plates seeded with OP-50 and allowed to recover for $\sim 24$ hours prior to imaging or lethality analysis.

\section{Lethality quantifications}

L4 worms were injected with indicated dsRNA and allowed to recover for 24 hours at $20^{\circ} \mathrm{C}$. 24 hours post-injection worms were then singled out and allowed to self-fertilize for an additional 24 hours. Worms were transferred to another plate for a final 24 hours, then disposed of. Plates corresponding to $24-48$ hours and $48-72$ hours post-injection were then scored for hatched larvae and unhatched embryos. Prior to counting, embryos were given 24 hours to hatch. The total number of embryos and larvae were combined for each time window to calculate embryonic lethality and brood size. A similar approach was used for noninjected control worms and worms containing deletion alleles.

\section{Immunoblots}

Generation of whole worm lysate

For a given condition, a microcentrifuge tube was filled with $30 \mu \mathrm{l}$ of M9 Buffer and the fill line was marked with a black marker. For each condition 35 adult worms were then placed in the microcentrifuge tube and washed three times with $M 9+0.1 \%$ Triton. After the final wash tubes were brought up to a final volume of $30 \mu \mathrm{l}$. Then, $10 \mu \mathrm{l}$ of $4 \mathrm{x}$ sample buffer was added and the tubes were mixed. The samples were then sonicated at $70^{\circ} \mathrm{C}$ for 15 minutes, followed by incubation for five minutes at $95^{\circ} \mathrm{C}$. Samples were re-sonicated at $70^{\circ} \mathrm{C}$ for an additional 15 minutes. Worm lysates were stored at $-20^{\circ} \mathrm{C}$ until they were run on an SDS-PAGE protein gel. 
Protein gel electrophoresis and antibody probing

For all protein gels, homemade $8 \%-10 \%$ SDS-PAGE were used. Worm lysates were reboiled at $95^{\circ} \mathrm{C}$ for five minutes and then $20 \mu \mathrm{l}(\sim 17.5$ worms) were loaded into each lane. The protein gel was then run at $80 \mathrm{~V}$ for 15 minutes to fully collapse samples. The protein gel was then run at $120 \mathrm{~V}$ for approximately 90 minutes, or until the sample buffer reached the bottom of the gel. Protein samples were then transferred overnight (16 hours at $4^{\circ} \mathrm{C}$ ) to an nitrocellulose membrane at $100 \mathrm{~mA}$. Membranes were blocked in fresh PBST for one hour at room temperature; membranes were then cut based on size and incubated overnight with the following primary antibodies: $1 \mu \mathrm{g} / \mathrm{mL}$ mouse $\alpha$-alpha-tubulin (EMD Millipore), $1 \mu \mathrm{g} / \mathrm{mL}$ mouse mAb414 (Biolegend), rabbit $\alpha-N P P-5 / N u p 107$ (1:300), rabbit $\alpha-N P P-10 N / N u p 98$ (1:500), rabbit $\alpha-N P P-10 C / N u p 96$ (1:500), and rabbit $\alpha-N P P-19 / N u p 53$ (1:1000).

Membranes were then rinsed three times quickly with PBST followed by four five-minute washes. Membranes were then incubated with appropriate secondary antibodies for one hour at room temperature. Secondary antibodies were diluted 1:5000 for horseradish peroxidase (HRP)conjugated goat-anti-rabbit and 1:7000 for HRP-conjugated goat-anti-mouse (Thermo Fischer Scientific). Membranes were rinsed and washed as described above. Prior to image acquisition membranes were incubated with Clarity Max Western ECL Substrate (BIO-RAD) for four minutes and then excess reagent was removed.

\section{Immunofluorescence}

Slide preparation

Microscope slides (Fisher Scientific Premium Microscope Slides Superfrost) were coated with $0.1 \%$ polylysine and dried on a low temperature heat-block. Slides were then baked at $95{ }^{\circ} \mathrm{C}$ for 30 minutes. Slides were used the same day that they were baked.

Fixation and primary antibody incubation

15-20 adult worms were picked into a $4 \mu \mathrm{l}$ drop of $\mathrm{ddH}_{2} \mathrm{O}$ and covered with a standard $18 \times 18 \mathrm{~mm}$ coverslip. Embryos were pushed out of the adult worms by pressing down on the 
corners of the coverslip with a pipet tip. To crack the eggshell and permeabilize the embryos, slides were placed in liquid nitrogen for five-minutes. Coverslips were quickly removed by using a razor blade to pop off the coverslip. Slides were then fixed in pre-chilled $100 \%$ methanol at -20 ${ }^{\circ} \mathrm{C}$ for 20 minutes. Following fixation slides were washed two times in $1 \mathrm{X}$ PBS $+0.2 \%$ Tween 20 (PBST) at room temperature for 10 minutes each. After the second wash, samples were blocked with $50 \mu \mathrm{L}$ of $1 \%$ BSA in PBST per slide in a humid chamber for one hour at $20^{\circ} \mathrm{C}$. Slides were then incubated overnight at $4^{\circ} \mathrm{C}$ with primary antibodies diluted in PBST (45 $\mu$ l per slide; rabbit $\alpha$ LMN1, $1 \mu \mathrm{g} / \mathrm{mL}$, mouse mAb414 $2.5 \mu \mathrm{g} / \mathrm{mL}$, rabbit $\alpha$-NPP-5/Nup107 (1:300), rabbit $\alpha-N P P$ 19/Nup53 (1:300), and rabbit a-MEL-28/Elys (1:500).

\section{Secondary antibody incubation and DAPI +Hoechst staining}

After overnight primary antibody incubation, slides were washed two times in $(50 \mu \mathrm{l}$ per slide) PBST at room temperature for 10 minutes each. Following the second wash, slides were incubated at $20^{\circ} \mathrm{C}$ for one hour in the dark with secondary antibodies diluted in PBST (45 $\mu$ l per slide, anti-rabbit Cy3/Rhodamine, 1:200; anti-mouse FITC, 1:200; [Jackson Immunoresearch]). Slides were again washed two times in $(50 \mu \mathrm{l}$ per slide) PBST at room temperature for 10 minutes each in the dark. Samples were stained with $1 \mu \mathrm{g} / \mathrm{mL}$ Hoechst (diluted from a $1 \mathrm{mg} / \mathrm{mL}$ stock in $\left.\mathrm{H}_{2} \mathrm{O}\right)$. Slides were washed two final times in $(50 \mu$ l per slide) PBST at room temperature for 10 minutes each in the dark. Finally, mounting media (Molecular Probes ProLong Gold Antifade Reagent with DAPI) was added to each sample. Coverslips were gently placed onto the slides and adhered with clear nail-polish. Slides were allowed to dry at $20^{\circ} \mathrm{C}$ and then stored at $-20^{\circ} \mathrm{C}$ until they were imaged.

\section{Image acquisition}

\section{Live microscopy}

First, $2 \%$ agarose imaging pads were made using molten agarose $\left(95^{\circ} \mathrm{C}\right)$ on a glass slide. Adult hermaphrodites were then dissected on glass slides and the embryos were transferred to 
two percent agarose imaging pads using a mouth pipette at $\left(\sim 20^{\circ} \mathrm{C}\right)$. Embryos were organized using an eyelash tool to group similar stage embryos. Images were acquired every 20 seconds. Five z-slices were taken for each time point with 2- $\mu \mathrm{m}$ steps. Images were acquired on an inverted Nikon Ti microscope equipped with a confocal scanner unit (CSU-XI, Yokogawa). Two solid state lasers (100-mW 488-nm and 50-mW 561-nm) were used in conjunction with a 60x objective lens (Å 1.4 NA pan Apo). Images were recorded with a high-resolution ORCA R-3 Digital CCD Camera (Hamamatsu).

Fluorescece recovery after photobleaching (FRAP)

First, $2 \%$ agarose imaging pads were made using molten agarose $\left(95^{\circ} \mathrm{C}\right)$ on a glass slide. Adult hermaphrodites were then dissected on glass slides and two-cell stage embryos were transferred to two percent agarose imaging pads using a mouth pipette at $\left(\sim 20^{\circ} \mathrm{C}\right)$. Embryos were organized using an eyelash tool to group similar stage embryos. A stimulation ROI was then drawn on a region of the nuclear envelope of an $A B$ nucleus (two cell stage embryo). Three images were taken prior to stimulation/bleaching of the ROI by a $100 \mathrm{nW}, 405 \mathrm{~nm}$ laser. Images were then acquired for the remainder of the cell cycle, until nuclear envelope breakdown. Images were taken every 10 s with 1 z-slice taken per time point.

Fixed microscopy

Immunofluorescent images were acquired on an inverted Nikon Ti Eclipse microscope. This microscope was equipped with solid state $100 \mathrm{~mW} \mathrm{405,} \mathrm{488,} \mathrm{514,} \mathrm{594,} \mathrm{561,} \mathrm{594,} \mathrm{and} 640$ nm lasers, a Yokogawa CSU-W1 confocal scanner unit, a 60x 1.4 NA plan Apo objective lens, and a prime BSI sCMOS camera.

qPCR

For each strain (10-15 medium plates) unsynchronized populations of adult worms were washed with 1x M9 buffer into 15-ml conical tubes. Worms were washed with five $\mathrm{mL}$ of M9, three times. After the final wash, the buffer was removed from the worm pellet and the samples were frozen at $-80^{\circ} \mathrm{C}$. Next an approximate $100 \mu \mathrm{l}$ worm pellet was ground using a motorized micro 
pestle. $1 \mathrm{~mL}$ of Trizol was then added to the ground worm pellet and the mixture was vortexed for 15 minutes at room temperature. Following vortexing, $200 \mu$ l of chloroform was added to the sample. The trizol-chloroform mixture was then briefly vortexed (15s) followed by an incubation at room temperature for five minutes. Next the solution was spun for 15 minutes at $4^{\circ} \mathrm{C}(12,000$ rpm). To extract the total RNA, the upper aqueous layer was removed (approximately $500 \mu l$ ) and transferred to a new microcentrifuge tube. $500 \mu \mathrm{l}$ of isopropanol was added to the tube and it was inverted six times.

To precipitate the RNA, the solution was incubated at room temperature for 10 minutes followed by a $10-$ minute spin $4^{\circ} \mathrm{C}(12,000 \mathrm{rpm})$. The supernatant was discarded, and the pellet was washed in $1 \mathrm{~mL}$ of $75 \%$ ethanol by inverting once and vortexing for 10 s. To pellet the RNA, the solution was spun for 5 minutes at $4^{\circ} \mathrm{C}(7500 \mathrm{rpm})$, the supernatant was removed, and the pellet was air-dried at room temperature for 5 minutes. The pellet was resuspended in $87 \mu$ of RNase-free water and incubated at $37^{\circ} \mathrm{C}$ for 15 minutes. After incubation, the isolated RNA was mixed with $10 \mu \mathrm{l}$ NEB Buffer $4,1 \mu \mathrm{l}$ of $50 \mu \mathrm{M} \mathrm{CaCl}$, and $2 \mu \mathrm{l}$ Ambion Turbo DNase and incubated at room temperature for 15 minutes. The RNA/DNase reaction was cleaned with a phenolchloroform extraction and eluted in $20 \mu \mathrm{l}$ of RNase free water. 500ng of total RNA was reverse transcribed using Invitrogen Super- Script II Reverse Transcriptase kit. The cDNA was stored at $-20^{\circ} \mathrm{C}$.

For qPCR reactions, cDNA ( $1200 \mathrm{ng} / \mu \mathrm{l})$ was diluted fivefold and pre-pared for amplification using a BioRad SYBR Green Supermix Kit with the primers for nucleoporin genes listed in key resource table as well as $h x k-2$ primers from [35]. For each biological replicate (two wildtype and two ndc1 1 ), four technical replicates were performed. Reactions were loaded into a 384-well plate for amplification. The reactions were amplified, and Ct values measured using a BioRad CFX 384 qPCR machine. An annealing temperature of $55^{\circ} \mathrm{C}$ was used for all genes. After amplification, Ct values for each nucleoporin gene were normalized to $h x k-2$, and the fold change relative to the control was calculated using the $2-(\Delta \Delta C t)$ method (BioRad). 


\section{Transmission Electron Microscopy}

\section{Sample preparation}

Wild-type N2 and SBW83 C. elegans hermaphrodites were dissected in M9 buffer and single embryos early in mitosis were selected and transferred to cellulose capillary tubes (Leica Microsystems, Vienna, Austria) with an inner diameter of $200 \mu \mathrm{m}$. The embryos were observed with a stereomicroscope until cleavage furrow ingression in late anaphase and then immediately cryo-immobilized using a LEICA ICE high-pressure freezer (Leica Microsystems, Vienna, Austria). Freeze substitution was performed over $3 \mathrm{~d}$ at $-90{ }^{\circ} \mathrm{C}$ in anhydrous acetone containing $1 \% \mathrm{OsO}_{4}$ and $0.1 \%$ uranyl acetate using an automatic freeze substitution machine (EM AFS, Leica Microsystems, Vienna, Austria). Epon/Araldite infiltrated samples were flat embedded in a thin layer of resin, polymerised for $2 \mathrm{~d}$ at $60^{\circ} \mathrm{C}$, and selected by light microscopy for re-mounting on dummy blocks. Serial semi-thick sections $(200 \mathrm{~nm})$ were cut using an Leica Ultracut S Microtome (Leica Microsystems, Vienna, Austria). Sections were collected on Pioloform-coated copper slot grids and poststained with $2 \%$ uranyl acetate in $70 \%$ methanol followed by Reynold's lead citrate.

Data acquisition by electron tomography

Colloidal gold particles (15 nm; Sigma-Aldrich) were attached to both sides of semi-thick sections collected on copper slot grids to serve as fiducial markers for subsequent image alignment. For dual-axis electron tomography, series of tilted views were recorded using an F20 electron microscopy (Thermo-Fisher, formerly FEI) operating at $200 \mathrm{kV}$ at magnifications ranging from [5000x-6500x], and recorded on a Gatan US4000 (4000px X 4000px) CCD or a Teitz TVIPS XF416 camera. Images were captured every $1.0^{\circ}$ over a $\pm 60^{\circ}$ range.

\section{$3 D$ reconstruction and automatic segmentation of microtubules}

We used the IMOD software package (http://bio3d.colourado.edu/imod), which contains all of the programs needed for calculating electron tomograms. For image processing the tilted views were aligned using the positions of the colloidal gold particles as fiducial markers. 
Tomograms were computed for each tilt axis using the R-weighted back-projection algorithm.

Nuclear envelope and NPC segmentation and measurement

The IMOD software package was used to segment the nuclear envelope and nuclear pore complexes in cntrl and ndc1 1 tomograms. Regions of continuous nuclear membranes were traced in the "non-core" region of the reforming nuclear envelope. Three criteria were used to distinguish nascent NPCs from simple NE holes, 1) the gaps between the two membrane edges were less than $100 \mathrm{~nm}, 2)$ the two membrane edges tapered to a point suggesting there was fusion of the inner and outer NE, and 3) there was stretches of continuous membranes above and below the gap.

\section{QUANTIFICATION AND STATISTICAL ANALYSIS}

\section{Image analysis}

\section{Nuclear import analysis}

To determine the fluorescence intensity of NLS-Lacl::GFP inside the nucleus of one- and two-cell stage embryos, the chromatin was traced with either the freehand or circle tool in FIJI . Camera background was determined by drawing a 50x50 pixel box in vacant areas of the video. Average cytoplasmic values were determined by drawing a $20 \times 20$ pixel box inside the embryo. The nuclear to cytoplasmic ratio $(\mathrm{N}: \mathrm{C})$ was determined by subtracting the average camera background from each value and then the nuclear value was divided by the cytoplasmic value. To account for differences in nuclear size, this ratio was then multiplied by the nuclear area. This process was repeated for each time point and condition.

\section{Line scan analysis of two-cell stage embryos}

A three-pixel wide by 10 -micron long line was drawn and centered on the nucleus to determine the fluorescence intensity. The line was then redrawn perpendicular to the first and a second measurement was taken. The values from these two lines were then averaged to give the fluorescence intensity of the nuclear envelope. The average value of the first and last 2 points of 
each line were used to subtract background from the rest of the line. These values were then plotted against the relative position along the line.

Line scan analysis of immunofluorescent images

A three-pixel wide by five-micron long line was drawn and centered on the nuclear envelope. The line was then redrawn perpendicular to the first and a second measurement was taken. The values from these two lines were then averaged to give the fluorescence intensity of the nuclear envelope. The same line scan was relocated to a clear area of the video to get the average intensity for camera background. Finally, to account for wide differences in fluorescence intensity, the data was normalized by dividing each value by the maximum fluorescence intensity. The final fluorescent intensities were then plotted against the relative position along the line. Additionally, the normalized value at the nuclear envelope was divided by the first five values on the line to determine the NE:cytoplasmic ratio (shown in Figure $\mathrm{xx}$ ) or by the last five values to determine the NE:nucleoplasmic ratio (shown in Figure $X X$ ).

Quantification of Nup160:GFP Puncta and colocalization with Ndc1:mRuby

To determine the number of NPP::GFP puncta/aggregates in the cytoplasm of one-cell stage embryos, maximum projections were generated using FIJI. The puncta were then tracked using the TrackMate plugin in FIJI [39]. This process was repeated to determine the number of puncta in ndc1, npp-7 RNAi depleted embryos. To determine the colocalization between the Nup160:GFP puncta and Ndc1:mRuby puncta, three-pixel wide line scans were drawn in each channel and plots were overlayed

FRAP analysis:

To evaluate the amount of recovery for Nup160:GFP and Ndc1:mNG, a rectangular ROI was used to measure the average fluorescence intensity of the nuclear envelope before and after photobleaching. These values were normalized to the pre-bleach frame and graphed relative to bleaching. To calculate the mobile/immobile fraction as well as the $T 1 / 2$, the data was fit to the 
exponential equation $f(t)=A^{*}\left(1-\exp \left(-\operatorname{tau}{ }^{*}\right.\right.$ Time $)$. To obtain the best fit, the parameters were fitted to the data using nonlinear least squares in R-studio.

\section{Statistical Analysis}

All data points are reported in graphs and error bar types are noted in figure legends. Statistical analysis was performed on datasets with multiple samples and independent biological repeats. The type of test used, sample sizes, and $\mathrm{P}$ values are reported in figure legends or in text $(P<0.05$ defined as significant). Statistical tests were performed using GraphPad Prism. 


\section{References}

1. Hampoelz, B., Andres-Pons, A., Kastritis, P., and Beck, M. (2019). Structure and Assembly of the Nuclear Pore Complex. Annu Rev Biophys 48, 515-536.

2. Lin, D.H., and Hoelz, A. (2019). The Structure of the Nuclear Pore Complex (An Update). Annu Rev Biochem 88, 725-783.

3. Dultz, E., Zanin, E., Wurzenberger, C., Braun, M., Rabut, G., Sironi, L., and Ellenberg, J. (2008). Systematic kinetic analysis of mitotic dis- and reassembly of the nuclear pore in living cells. The Journal of cell biology 180, 857-865.

4. Otsuka, S., and Ellenberg, J. (2018). Mechanisms of nuclear pore complex assembly two different ways of building one molecular machine. FEBS Lett 592, 475-488.

5. Otsuka, S., Steyer, A.M., Schorb, M., Hériché, J.K., Hossain, M.J., Sethi, S., Kueblbeck, M., Schwab, Y., Beck, M., and Ellenberg, J. (2018). Postmitotic nuclear pore assembly proceeds by radial dilation of small membrane openings. Nat Struct Mol Biol 25, 21-28.

6. Walther, T.C., Alves, A., Pickersgill, H., Loïodice, I., Hetzer, M., Galy, V., Hülsmann, B.B., Köcher, T., Wilm, M., Allen, T., et al. (2003). The conserved Nup107-160 complex is critical for nuclear pore complex assembly. Cell 113, 195-206.

7. Eisenhardt, N., Redolfi, J., and Antonin, W. (2014). Interaction of Nup53 with Ndc1 and Nup155 is required for nuclear pore complex assembly. J Cell Sci 127, 908-921.

8. Mansfeld, J., Guttinger, S., Hawryluk-Gara, L.A., Pante, N., Mall, M., Galy, V., Haselmann, U., Muhlhausser, P., Wozniak, R.W., Mattaj, I.W., et al. (2006). The conserved transmembrane nucleoporin NDC1 is required for nuclear pore complex assembly in vertebrate cells. Molecular cell 22, 93-103.

9. Onischenko, E., Stanton, L.H., Madrid, A.S., Kieselbach, T., and Weis, K. (2009). Role of the Ndc1 interaction network in yeast nuclear pore complex assembly and maintenance. The Journal of cell biology 185, 475-491.

10. Sönnichsen, B., Koski, L.B., Walsh, A., Marschall, P., Neumann, B., Brehm, M., Alleaume, A.M., Artelt, J., Bettencourt, P., Cassin, E., et al. (2005). Full-genome RNAi profiling of early embryogenesis in Caenorhabditis elegans. Nature 434, 462-469.

11. Stavru, F., Hulsmann, B.B., Spang, A., Hartmann, E., Cordes, V.C., and Gorlich, D. (2006). NDC1: a crucial membrane-integral nucleoporin of metazoan nuclear pore complexes. The Journal of cell biology 173, 509-519.

12. Kume, K., Cantwell, H., Neumann, F.R., Jones, A.W., Snijders, A.P., and Nurse, P. (2017). A systematic genomic screen implicates nucleocytoplasmic transport and membrane growth in nuclear size control. PLoS genetics 13, e1006767.

13. Ladouceur, A.M., Dorn, J.F., and Maddox, P.S. (2015). Mitotic chromosome length scales in response to both cell and nuclear size. The Journal of cell biology 209, 645-651.

14. Levy, D.L., and Heald, R. (2010). Nuclear size is regulated by importin alpha and Ntf2 in Xenopus. Cell 143, 288-298.

15. Levy, D.L., and Heald, R. (2012). Mechanisms of intracellular scaling. Annual review of cell and developmental biology 28, 113-135.

16. Penfield, L., Shankar, R., Szentgyorgyi, E., Laffitte, A., Mauro, M.S., Audhya, A., MullerReichert, T., and Bahmanyar, S. (2020). Regulated lipid synthesis and LEM2/CHMP7 jointly control nuclear envelope closure. The Journal of cell biology 219.

17. Oegema, K., and Hyman, A.A. (2006). Cell division. WormBook : the online review of C. elegans biology, 1-40.

18. Galy, V., Mattaj, I.W., and Askjaer, P. (2003). Caenorhabditis elegans nucleoporins Nup93 and Nup205 determine the limit of nuclear pore complex size exclusion in vivo. Mol Biol Cell 14, 5104-5115. 
19. Ródenas, E., Klerkx, E.P., Ayuso, C., Audhya, A., and Askjaer, P. (2009). Early embryonic requirement for nucleoporin Nup35/NPP-19 in nuclear assembly. Developmental biology 327, 399-409.

20. Vollmer, B., Lorenz, M., Moreno-Andrés, D., Bodenhöfer, M., De Magistris, P., Astrinidis, S.A., Schooley, A., Flötenmeyer, M., Leptihn, S., and Antonin, W. (2015). Nup153 Recruits the Nup107-160 Complex to the Inner Nuclear Membrane for Interphasic Nuclear Pore Complex Assembly. Dev Cell 33, 717-728.

21. Vollmer, B., Schooley, A., Sachdev, R., Eisenhardt, N., Schneider, A.M., Sieverding, C., Madlung, J., Gerken, U., Macek, B., and Antonin, W. (2012). Dimerization and direct membrane interaction of Nup53 contribute to nuclear pore complex assembly. Embo j 31 , 4072-4084.

22. Walther, T.C., Fornerod, M., Pickersgill, H., Goldberg, M., Allen, T.D., and Mattaj, I.W. (2001). The nucleoporin Nup153 is required for nuclear pore basket formation, nuclear pore complex anchoring and import of a subset of nuclear proteins. Embo j 20, 5703-5714.

23. Liu, S., and Pellman, D. (2020). The coordination of nuclear envelope assembly and chromosome segregation in metazoans. Nucleus 11, 35-52.

24. Lu, L., Ladinsky, M.S., and Kirchhausen, T. (2011). Formation of the postmitotic nuclear envelope from extended ER cisternae precedes nuclear pore assembly. The Journal of cell biology 194, 425-440.

25. Hattersley, N., Cheerambathur, D., Moyle, M., Stefanutti, M., Richardson, A., Lee, K.Y., Dumont, J., Oegema, K., and Desai, A. (2016). A Nucleoporin Docks Protein Phosphatase 1 to Direct Meiotic Chromosome Segregation and Nuclear Assembly. Dev Cell 38, 463477.

26. Hampoelz, B., Mackmull, M.T., Machado, P., Ronchi, P., Bui, K.H., Schieber, N., Santarella-Mellwig, R., Necakov, A., Andrés-Pons, A., Philippe, J.M., et al. (2016). Preassembled Nuclear Pores Insert into the Nuclear Envelope during Early Development. Cell 166, 664-678.

27. Galy, V., Askjaer, P., Franz, C., López-Iglesias, C., and Mattaj, I.W. (2006). MEL-28, a novel nuclear-envelope and kinetochore protein essential for zygotic nuclear-envelope assembly in C. elegans. Current biology : CB 16, 1748-1756.

28. Ródenas, E., González-Aguilera, C., Ayuso, C., and Askjaer, P. (2012). Dissection of the NUP107 nuclear pore subcomplex reveals a novel interaction with spindle assembly checkpoint protein MAD1 in Caenorhabditis elegans. Mol Biol Cell 23, 930-944.

29. Daigle, N., Beaudouin, J., Hartnell, L., Imreh, G., Hallberg, E., Lippincott-Schwartz, J., and Ellenberg, J. (2001). Nuclear pore complexes form immobile networks and have a very low turnover in live mammalian cells. The Journal of cell biology 154, 71-84.

30. Onischenko, E., Noor, E., Fischer, J.S., Gillet, L., Wojtynek, M., Vallotton, P., and Weis, K. (2020). Maturation Kinetics of a Multiprotein Complex Revealed by Metabolic Labeling. Cell 183, 1785-1800.e1726.

31. Linder, M.I., Köhler, M., Boersema, P., Weberruss, M., Wandke, C., Marino, J., Ashiono, C., Picotti, P., Antonin, W., and Kutay, U. (2017). Mitotic Disassembly of Nuclear Pore Complexes Involves CDK1- and PLK1-Mediated Phosphorylation of Key Interconnecting Nucleoporins. Dev Cell 43, 141-156.e147.

32. Marelli, M., Lusk, C.P., Chan, H., Aitchison, J.D., and Wozniak, R.W. (2001). A link between the synthesis of nucleoporins and the biogenesis of the nuclear envelope. The Journal of cell biology 153, 709-724.

33. Chou, Y.Y., Upadhyayula, S., Houser, J., He, K., Skillern, W., Scanavachi, G., Dang, S., Sanyal, A., Ohashi, K.G., Di Caprio, G., et al. (2021). Inherited nuclear pore substructures template post-mitotic pore assembly. Dev Cell 56, 1786-1803 e1789.

34. Penfield, L., Wysolmerski, B., Mauro, M., Farhadifar, R., Martinez, M.A., Biggs, R., Wu, H.Y., Broberg, C., Needleman, D., and Bahmanyar, S. (2018). Dynein pulling forces 
counteract lamin-mediated nuclear stability during nuclear envelope repair. Mol Biol Cell 29, 852-868.

35. Kudron, M., Niu, W., Lu, Z., Wang, G., Gerstein, M., Snyder, M., and Reinke, V. (2013). Tissue-specific direct targets of Caenorhabditis elegans Rb/E2F dictate distinct somatic and germline programs. Genome biology 14, R5.

36. Hastie, E., Sellers, R., Valan, B., and Sherwood, D.R. (2019). A Scalable CURE Using a CRISPR/Cas9 Fluorescent Protein Knock-In Strategy in Caenorhabditis elegans. J Microbiol Biol Educ 20.

37. Arribere, J.A., Bell, R.T., Fu, B.X., Artiles, K.L., Hartman, P.S., and Fire, A.Z. (2014). Efficient marker-free recovery of custom genetic modifications with CRISPR/Cas9 in Caenorhabditis elegans. Genetics 198, 837-846.

38. Paix, A., Folkmann, A., Rasoloson, D., and Seydoux, G. (2015). High Efficiency, Homology-Directed Genome Editing in Caenorhabditis elegans Using CRISPR-Cas9 Ribonucleoprotein Complexes. Genetics 201, 47-54.

39. Tinevez, J.Y., Perry, N., Schindelin, J., Hoopes, G.M., Reynolds, G.D., Laplantine, E., Bednarek, S.Y., Shorte, S.L., and Eliceiri, K.W. (2017). TrackMate: An open and extensible platform for single-particle tracking. Methods 115, 80-90. 